\title{
Convolution of radius functions on $\mathbb{R}^{3}$
}

\author{
by Konstanty Holly (Kraków)
}

\begin{abstract}
We reduce the convolution of radius functions to that of 1-variable functions. Then we present formulas for computing convolutions of an abstract radius function on $\mathbb{R}^{3}$ with various integral kernels - given by elementary or discontinuous functions. We also prove a theorem on the asymptotic behaviour of a convolution at infinity. Lastly, we deduce some estimates which enable us to find the asymptotics of the velocity and pressure of a fluid (described by the Navier-Stokes equations) in the boundary layer.
\end{abstract}

1. Reduction of the convolution of radius functions on $\mathbb{R}^{3}$ to a convolution on $\mathbb{R}^{1}$. The convolution of Borel measurable functions $f, g$ : $\mathbb{R}^{n} \rightarrow[0, \infty]$ is the function

$$
f * g: \mathbb{R}^{n} \ni x \mapsto \int_{\mathbb{R}^{n}} f(x-y) g(y) d y \in[0, \infty],
$$

where $d y$ denotes the Lebesgue measure on $\mathbb{R}^{n}$.

(1.1) Example. For $a, b>0$,

$$
\left(\frac{a}{\pi}\right)^{n / 2} e^{-a|\cdot|^{2}} *\left(\frac{b}{\pi}\right)^{n / 2} e^{-b|\cdot|^{2}}=\left(\frac{1}{\pi} \frac{a b}{a+b}\right)^{n / 2} e^{-(a b /(a+b))|\cdot|^{2}} .
$$

(1.3) Example. For $\lambda \in] 0, n[$ consider the hyperboloid

$$
K_{\lambda}: \mathbb{R}^{n} \ni x \mapsto|x|^{-\lambda} \in[0, \infty] .
$$

If $0<\alpha, \beta<n$ and $\alpha+\beta>n$, then

$$
C(n, \alpha) K_{\alpha} * C(n, \beta) K_{\beta}=C(n, \alpha+\beta-n) K_{\alpha+\beta-n},
$$

where $C(n, \lambda):=2^{\lambda}(4 \pi)^{-n / 2} \Gamma(\lambda / 2) \Gamma((n-\lambda) / 2)^{-1}$. Identity (1.4) is known as the law of composition of the M. Riesz kernels (see [4] and [3]).

The proofs of (1.2) and of Theorem (1.6) are included in Section 1'. Section $2^{\prime}$ contains the proofs of the theorems from Section 2, etc.

1991 Mathematics Subject Classification: Primary 26B20.

Key words and phrases: integral formulas, asymptotic behaviour of convolution at $\infty$. 
K. Holly

(1.5) From now on it is assumed that all Borel measurable functions which are integrated have separable ranges.

Consider Banach spaces $W_{1}, W_{2}, W$ over a number field $K(\in\{\mathbb{R}, \mathbb{C}\})$ and a continuous $K$-bilinear operator $W_{1} \times W_{2} \ni\left(v_{1}, v_{2}\right) \mapsto v_{1} v_{2} \in W$. The convolution of Borel measurable functions $f_{1}: \mathbb{R}^{n} \rightarrow W_{1}, f_{2}: \mathbb{R}^{n} \rightarrow W_{2}$ is the function

$$
\left(f_{1} * f_{2}\right)(x):=\int_{\mathbb{R}^{n}} f_{1}(x-y) f_{2}(y) d y
$$

with values in $W$, defined on

$$
\operatorname{dom}\left(f_{1} * f_{2}\right):=\left\{x \in \mathbb{R}^{n}:\left(\left|f_{1}\right| *\left|f_{2}\right|\right)(x)<\infty\right\} .
$$

A function of the form

$$
\mathbb{R}^{n} \ni x \mapsto \kappa(|x|) \in W,
$$

where $\kappa: \mathbb{R}_{+} \rightarrow W$, is called a radius function. The functions mentioned in Examples (1.1), (1.3) are such functions.

(1.6) TheOREM. Consider Borel measurable functions $w_{i}: \mathbb{R} \rightarrow W_{i}$, $i=1,2$, such that $w_{1}$ is odd, while $w_{2}$ is even and absolutely continuous. Fix $x \in \mathbb{R}^{3}$. Then

$$
\left(w_{1} * w_{2}\right)(|x|)=\frac{|x|}{2 \pi}\left(-\frac{w_{1}(|\cdot|)}{|\cdot|} * \frac{\dot{w}_{2}(|\cdot|)}{|\cdot|}\right)(x)
$$

provided

$$
\left(\left|w_{1}\right| *\left|w_{2}\right|\right)(|x|)<\infty \quad \text { and } \quad\left(\left|\frac{w_{1}(|\cdot|)}{|\cdot|}\right| *\left|\frac{\dot{w}_{2}(|\cdot|)}{|\cdot|}\right|\right)(x)<\infty .
$$

In practice the functions $f_{i}: \mathbb{R}^{3} \rightarrow W_{i}(i=1,2)$ are usually given and we look for functions $w_{i}: \mathbb{R} \rightarrow W_{i}(i=1,2)$ such that

$$
f_{1}=-\frac{w_{1}(|\cdot|)}{|\cdot|}, \quad f_{2}=\frac{\dot{w}_{2}(|\cdot|)}{|\cdot|} .
$$

We do not know any analogue of Theorem (1.6) for $\mathbb{R}^{n}, n \neq 3$. For instance, for $n=2$ on the right-hand side of the analogue of $\left(1^{\prime} .5\right)$ there appears the Bessel function $J_{0}$, which excludes any further analogies with our reasoning.

2. Various applications of Theorem (1.6). First, we will show that the boundary value problem $\{\Delta u=\kappa(|\cdot|), u(\infty)=0\}$ in $\mathbb{R}^{3}$ may be reduced to the study of a one-dimensional integral.

(2.1) REMARK. Let $\kappa:] 0, \infty[\rightarrow W$ be a Borel measurable function such that $\int_{0}^{1} r^{2}|\kappa(r)| d r<\infty$ and $\int_{1}^{\infty} r|\kappa(r)| d r<\infty$. Then for every $x \in \mathbb{R}^{3} \backslash\{0\}$ 
we have

$$
\left(\frac{1}{4 \pi|\cdot|} * \kappa(|\cdot|)\right)(x)=\frac{1}{|x|} \int_{0}^{|x|} r^{2} \kappa(r) d r+\int_{|x|}^{\infty} r \kappa(r) d r .
$$

This formula is "logically equivalent" to the formula from Corollary ( $\left.1^{\prime} .15\right)$ see Lemma $\left(1^{\prime} .11\right)$ and Digression $\left(1^{\prime} .15\right)^{*}$.

(2.2) Corollary. For all $c>0$ and $x \in \mathbb{R}^{3}$,

$$
\left(\frac{1}{4 \pi|\cdot|} * e^{-c|\cdot|^{2}}\right)(x)=\frac{1}{2 c} \int_{0}^{1} e^{-c|x|^{2} \tau^{2}} d \tau
$$

The convolution of the integral kernel $(-4 \pi|\cdot|)^{-1}$ of the stationary heat equation

$$
0=\Delta u+f
$$

in $\mathbb{R}^{3}$, with the kernel $(4 \pi \nu t)^{-3 / 2} \exp \left(-|\cdot|^{2} /(4 \nu t)\right)$ of the heat evolution equation

$$
\frac{\partial u}{\partial t}(t, \cdot)=\nu \Delta u(t, \cdot)+f(t, \cdot)
$$

appears in the integral kernel of the nonstationary Navier-Stokes equations in $\mathbb{R}^{3}$ — see (0.5) in [1]. Corollary (2.2) may also be derived from the following:

(2.3) REMARK. If $\kappa:] 0, \infty[\rightarrow[0, \infty]$ is a Borel measurable function and $c>0$, then for all $x \in \mathbb{R}^{3} \backslash\{0\}$,

$$
\frac{1}{4 \pi}\left(\kappa(|\cdot|) * e^{-(c / 2)|\cdot|^{2}}\right)(x)=e^{-(c / 2)|x|^{2}} \int_{0}^{\infty} \kappa(r) r^{2} e^{-(c / 2) r^{2}} \frac{\operatorname{sh}(c|x| r)}{c|x| r} d r .
$$

We now give a few formulas for the convolution of a radius function with the rational function $\left(a+|\cdot|^{2}\right)^{-m}(a>0, m \in \mathbb{N})$. Differentiation or integration with respect to the parameter $a$ sometimes enables us to obtain some formulas from simpler ones. For instance, for the relations given below we have

$$
\begin{gathered}
\frac{d}{d a}(2.5)=(2.7) \Rightarrow(2.10), \quad \int(2.10) d b=(2.9), \\
\frac{d}{d a}(2.10)=(2.11), \quad \frac{d^{2}}{d a^{2}}(2.11)=(2.12) .
\end{gathered}
$$

(2.4) Lemma. Let $\kappa:] 0, \infty[\rightarrow W$ be a Borel measurable function such that $\int_{0}^{1} r^{2}|\kappa(r)| d r<\infty$ and $\int_{1}^{\infty}|\kappa(r)| d r<\infty$. Then for all $x \in \mathbb{R}^{3} \backslash\{0\}$,

$$
\frac{1}{\pi}\left(\frac{1}{a+|\cdot|^{2}} * \kappa(|\cdot|)\right)(x)=\frac{1}{|x|} \int_{0}^{\infty} r\left(\ln \frac{a+(r+|x|)^{2}}{a+(r-|x|)^{2}}\right) \kappa(r) d r .
$$


(2.6) Corollary. If $\kappa:] 0, \infty[\rightarrow W$ is a Borel measurable function such that $\int_{0}^{1} r^{2}|\kappa(r)| d r<\infty$ and $\int_{1}^{\infty} r^{-2}|\kappa(r)| d r<\infty$, then for all $x \in \mathbb{R}^{3}$,

(2.7) $\frac{1}{4 \pi}\left(\frac{1}{\left(a+|\cdot|^{2}\right)^{2}} * \kappa(|\cdot|)\right)(x)=\int_{0}^{\infty} \frac{r^{2} \kappa(r) d r}{\left(a+(r-|x|)^{2}\right)\left(a+(r+|x|)^{2}\right)}$.

(2.8) Corollary. Let $a, b>0$, and let $*$ denote convolution in $\mathbb{R}^{3}$. Then

$$
\frac{1}{a^{2}+|\cdot|^{2}} * \frac{1}{b^{2}+|\cdot|^{2}}=\frac{2 \pi^{2}}{|\cdot|} \arctan \frac{|\cdot|}{a+b}
$$

$($ for $x=0$ see $(3.11)$ and $(3.12))$,

$$
\begin{aligned}
& \frac{1}{a^{2}+|\cdot|^{2}} * \frac{1}{\left(b^{2}+|\cdot|^{2}\right)^{2}}=\frac{\pi^{2}}{b} \frac{1}{(a+b)^{2}+|\cdot|^{2}} \\
& \left(\frac{\sqrt{a}}{\pi} \frac{1}{a^{2}+|\cdot|^{2}}\right)^{2} *\left(\frac{\sqrt{b}}{\pi} \frac{1}{b^{2}+|\cdot|^{2}}\right)^{2} \\
& =\left(\frac{\sqrt{a+b}}{\pi} \frac{1}{(a+b)^{2}+|\cdot|^{2}}\right)^{2} \\
& \left(\frac{1}{a^{2}+|\cdot|^{2}}\right)^{4} *\left(\frac{1}{b^{2}+|\cdot|^{2}}\right)^{2}=\frac{\pi^{2}}{a^{3}}\left(\frac{1}{(a+b)^{2}+|\cdot|^{2}}\right)^{2} \\
& \times\left(\frac{1}{8 a^{2}}+\frac{a+b}{2 a} \frac{1}{(a+b)^{2}+|\cdot|^{2}}+\frac{(a+b)^{3}}{b}\left(\frac{1}{(a+b)^{2}+|\cdot|^{2}}\right)^{2}\right) .
\end{aligned}
$$

From the point of view of symmetry, (2.11) resembles the formulas (1.2), (1.4).

From now on we compute the convolution of a radius function with specific discontinuous functions.

(2.13) Remark. Fix $0<\varrho<\infty$. Let $\chi_{\varrho}$ denote the characteristic function of the interval $[-\varrho, \varrho]$. If $\kappa \in L_{\mathrm{loc}}^{1}(] 0, \infty[, W)$ and $\int_{0}^{1} r|\kappa(r)| d r<\infty$, then for all $x \in \mathbb{R}^{3} \backslash\{0\}$,

$$
\begin{aligned}
\left(\chi_{\varrho}(|\cdot|) * \kappa(|\cdot|)\right)(x) & =\frac{\pi}{|x|} \int_{|x|-\varrho}^{|x|+\varrho}\left(\varrho^{2}-|r-| x||^{2}\right) r \kappa(|r|) d r, \\
\left(\left(|\cdot| \chi_{\varrho}(|\cdot|)\right) * \kappa(|\cdot|)\right)(x) & =\frac{2 \pi}{3|x|} \int_{|x|-\varrho}^{|x|+\varrho}\left(\varrho^{3}-|r-| x||^{3}\right) r \kappa(|r|) d r .
\end{aligned}
$$

(2.14) Remark. Assume that $0<\varrho<\infty, 3<\lambda<\infty$ and let $\kappa$ : ] $0, \infty\left[\rightarrow W\right.$ be a Borel measurable function such that $\int_{0}^{1} r|\kappa(r)| d r<\infty$ 
and $\int_{1}^{\infty} r^{3-\lambda}|\kappa(r)| d r<\infty$. Define

$$
f(r):= \begin{cases}0 & \text { if } r \leq \varrho, \\ r^{-\lambda} & \text { if } r>\varrho .\end{cases}
$$

Then for all $x \in \mathbb{R}^{3} \backslash\{0\}$,

$$
\begin{aligned}
& (f(|\cdot|) * \kappa(|\cdot|))(x) \\
= & \frac{2 \pi}{\lambda-2} \frac{1}{|x|}\left(\varrho^{2-\lambda} \int_{|r-| x||<\varrho} r \kappa(|r|) d r+\int_{|r-| x||>\varrho}|r-| x||^{2-\lambda} r \kappa(|r|) d r\right) .
\end{aligned}
$$

3. Asymptotic behaviour of a convolution as $|x| \rightarrow \infty$. We start with two estimates of a convolution that involves the function $(1+|\cdot|)^{-\gamma}$.

(3.1) Remark. Let $f: \mathbb{R}^{n} \rightarrow[0, \infty]$ be a Borel measurable function with compact support, i.e. $\varrho:=\sup _{f(x) \neq 0}|x|<\infty$. Then for all $\gamma \in \mathbb{R}$,

$$
\sup _{x \in \mathbb{R}^{n}}(1+|x|)^{\gamma}\left((1+|\cdot|)^{-\gamma} * f\right)(x) \leq(1+\varrho)^{|\gamma|}\|f\|_{L^{1}} .
$$

(3.3) Lemma. If $0 \leq \gamma \leq \lambda$ and $\lambda>3$ then

$$
\sup _{x \in \mathbb{R}^{3}}(1+|x|)^{\gamma}\left((1+|\cdot|)^{-\gamma} *(1+|\cdot|)^{-\lambda}\right)(x)<\infty .
$$

In spite of the resemblance between (3.2) and (3.4), Lemma (3.3) does not follow from Remark (3.1), because $\operatorname{supp}(1+|\cdot|)^{-\lambda}$ is unbounded.

The functions $\varphi$ and $w$ considered in (3.5) below are not necessarily radius functions.

(3.5) Theorem. Assume that $\lambda>3,0 \leq \gamma<\lambda, \varphi: \mathbb{R}^{3} \rightarrow W_{1}$, and $w: \mathbb{R}^{3} \rightarrow W_{2}$. Assume that

(3.6) the functions $(1+|\cdot|)^{\lambda} \varphi,(1+|\cdot|)^{\gamma} w$ are bounded and Borel measurable.

Moreover, suppose that

$$
\lim _{x \rightarrow \infty}\left(|x|^{\gamma} w(x)-w_{\infty}(x /|x|)\right)=0,
$$

where $w_{\infty}: S_{2} \rightarrow W_{2}$ is continuous. Then

$$
\lim _{x \rightarrow \infty}\left(|x|^{\gamma}(\varphi * w)(x)-\int_{\mathbb{R}^{3}} \varphi(y) d y \cdot w_{\infty}(x /|x|)\right)=0 .
$$

$(3.9)^{*}$ The need for such a theorem appeared in a natural way in the theory of the Navier-Stokes equations. It is used in the proof of a theorem (announced in [2]) on the asymptotic behaviour of the velocity $v(t, x)$ and pressure $p(t, x)$ of the free fluid in $\mathbb{R}^{3}$ as $|x| \rightarrow \infty$. 
When $\gamma=\lambda$, Theorem (3.5) is not true, as shown by the following example:

(3.10) Example. The functions

$$
\varphi=w:=\left(\frac{1}{\pi} \frac{1}{1+|\cdot|^{2}}\right)^{2}
$$

satisfy assumptions $(3.6),(3.7)$ for $\gamma=\lambda=4, w_{\infty} \equiv \pi^{-2}$. Note that

(3.11) the function $\frac{1}{1+|\cdot|^{2}} * \frac{1}{1+|\cdot|^{2}}: \mathbb{R}^{3} \rightarrow \mathbb{R}$ is continuous, since $1 /\left(1+|\cdot|^{2}\right) \in L^{2}\left(\mathbb{R}^{3}\right)$. Bearing that in mind and using (2.9) we compute:

$$
\begin{aligned}
\int_{\mathbb{R}^{3}} \varphi(y) d y & =\frac{1}{\pi^{2}} \int_{\mathbb{R}^{3}} \frac{d y}{\left(1+|y|^{2}\right)^{2}}=\frac{1}{\pi^{2}}\left(\frac{1}{1+|\cdot|^{2}} * \frac{1}{1+|\cdot|^{2}}\right)(0) \\
& =\frac{1}{\pi^{2}} \lim _{0 \neq x \rightarrow 0}\left(\frac{1}{1+|\cdot|^{2}} * \frac{1}{1+|\cdot|^{2}}\right)(x) \\
& =\frac{1}{\pi^{2}} \lim _{0 \neq x \rightarrow 0} \frac{2 \pi^{2}}{|x|} \arctan \frac{|x|}{2}=1 .
\end{aligned}
$$

For $a=b=1$ the formula (2.11) takes the form

$$
\varphi * w=\left(\frac{\sqrt{2}}{\pi} \frac{1}{4+|\cdot|^{2}}\right)^{2} .
$$

Finally,

$$
\begin{aligned}
\lim _{x \rightarrow \infty}|x|^{4}(\varphi * w)(x) & =\lim _{x \rightarrow \infty} \frac{2}{\pi^{2}} \frac{|x|^{4}}{\left(4+|x|^{2}\right)^{2}} \\
& =\frac{2}{\pi^{2}} \neq 1 \cdot \frac{1}{\pi^{2}} \equiv \int_{\mathbb{R}^{3}} \varphi(y) d y \cdot w_{\infty}
\end{aligned}
$$

4. Some special estimates. We present three inequalities needed in the proof of the theorem mentioned in Digression (3.9)*. From now on the parameters $a, \nu$ are fixed positive numbers.

(4.1) Lemma. Let $E:] 0, \infty\left[\times \mathbb{R}^{3} \rightarrow \mathbb{R}\right.$ be the heat kernel in $\mathbb{R}^{3}$, i.e.

$$
E(t, x):=(\sqrt{2 \nu t})^{-3} \exp \left(-\frac{1}{2}\left|\frac{x}{\sqrt{2 \nu t}}\right|^{2}\right) \text {. }
$$

Then for all $t>0$,

$$
(2 \pi)^{-3 / 2} \frac{1}{\left(a+|\cdot|^{2}\right)^{2}} * E(t, \cdot) \leq\left(1+\frac{3 \nu t}{2 a}\right) \frac{1}{\left(a+|\cdot|^{2}\right)^{2}} .
$$


(4.2) Lemma. Define

$$
F:] 0, \infty\left[\times \mathbb{R}^{3} \ni(\tau, x) \mapsto \begin{cases}(2 \nu \tau)^{-5 / 2}|x| & \text { if }|x|<\sqrt{2 \nu \tau} \\ 0 & \text { if }|x| \geq \sqrt{2 \nu \tau}\end{cases}\right.
$$

Then there exists an increasing continuous function $M: \mathbb{R}_{+} \rightarrow \mathbb{R}_{+}$(see $\left.\left(4^{\prime} .12\right)\right)$ such that for all $\tau>0$,

$$
\left(a+|\cdot|^{2}\right)^{-4} * F(\tau, \cdot) \leq(\nu \tau)^{-1 / 2} M(\nu \tau / a)\left(a+|\cdot|^{2}\right)^{-4} .
$$

(4.3) Lemma. Define

$$
G:] 0, \infty\left[\times \mathbb{R}^{3} \ni(\tau, x) \mapsto \begin{cases}0 & \text { if }|x|<\sqrt{2 \nu \tau} \\ |x|^{-4} & \text { if }|x| \geq \sqrt{2 \nu \tau}\end{cases}\right.
$$

Then there exist a constant $C \in \mathbb{R}_{+}\left(\right.$see $\left.\left(4^{\prime} .18\right)\right)$ and an increasing continuous function $M: \mathbb{R}_{+} \rightarrow \mathbb{R}_{+}$(see (4'.19)) such that for all $\tau>0$,

$\left(a+|\cdot|^{2}\right)^{-4} * G(\tau, \cdot) \leq C a^{-5 / 2}\left(a+|\cdot|^{2}\right)^{-2}+(\nu \tau)^{-1 / 2} M(\nu \tau / a)\left(a+|\cdot|^{2}\right)^{-4}$.

$\mathbf{1}^{\prime}$. Proofs. The Fourier transform of a summable function $u: \mathbb{R}^{n} \rightarrow W$ (in the case of $K=\mathbb{C}$ ) is the function $\widehat{u}=\mathcal{F} u: \mathbb{R}^{n} \rightarrow W$ given by

$$
\widehat{u}(\xi):=(2 \pi)^{-n / 2} \int_{\mathbb{R}^{n}} e^{-i x \xi} u(x) d x,
$$

where $x \xi=(x \mid \xi):=\sum_{k=1}^{n} x_{k} \xi_{k}$. Throughout Sections $1^{\prime}, 2^{\prime}, 3^{\prime}$ the following abbreviations are used:

$(\mathrm{L} \nearrow)=$ the Lebesgue monotone convergence theorem,

$(\overline{\mathrm{L}})=$ the Lebesgue dominated convergence theorem.

$\left(1^{\prime} .1\right)$ Proof of (1.2). It is known that $\widehat{\varphi}=\varphi$ for $\varphi:=e^{-(1 / 2)|\cdot|^{2}}$ $\left(\in S\left(\mathbb{R}^{n}, \mathbb{C}\right)\right)$. Let $h_{\lambda}$ stand for the homothety with scale $\lambda \in \mathbb{R} \backslash\{0\}$. Then of course $\left(\varphi \circ h_{\lambda}\right)^{\wedge}=|\lambda|^{-n} \widehat{\varphi} \circ h_{1 / \lambda}$. Therefore

$$
e^{-a|\cdot|^{2}}=\varphi \circ h_{\sqrt{2 a}}=(2 a)^{-n / 2}(\sqrt{2 a})^{n} \widehat{\varphi} \circ h_{\sqrt{2 a}}=(2 a)^{-n / 2}\left(\varphi \circ h_{\sqrt{1 /(2 a})}\right)^{\wedge} .
$$

Hence

$$
\begin{aligned}
e^{-a|\cdot|^{2} \widetilde{*} e^{-b|\cdot|^{2}}} & =(4 a b)^{-n / 2}\left(\varphi \circ h_{\sqrt{1 /(2 a)}}\right)^{\wedge} \widetilde{*}\left(\varphi \circ h_{\sqrt{1 /(2 b)}}\right)^{\wedge} \\
& =(4 a b)^{-n / 2}\left(\left(\varphi \circ h_{\sqrt{1 /(2 a)}}\right)\left(\varphi \circ h_{\sqrt{1 /(2 b)}}\right)\right)^{\wedge} \\
& =(4 a b)^{-n / 2}\left(\varphi \circ h_{\sqrt{(a+b) /(2 a b)}}\right)^{-n} \\
& =(4 a b)^{-n / 2}\left(\sqrt{\frac{a+b}{2 a b}}\right)^{-n} \widehat{\varphi} \circ h_{\sqrt{(2 a b) /(a+b)}} \\
& =(2(a+b))^{-n / 2} e^{-(a b /(a+b)))\left.\cdot\right|^{2}}
\end{aligned}
$$


where

$$
u \widetilde{*} v:=(2 \pi)^{-n / 2} u * v .
$$

$\left(1^{\prime} .3\right)$ Lemma. Assume that $K=\mathbb{C}$ and an even Borel measurable function $f: \mathbb{R} \rightarrow W$ satisfies $\int_{0}^{1} r|f(r)| d r<\infty$ and $\int_{1}^{\infty} r^{2}|f(r)| d r<\infty$. Then $f(|\cdot|) \in L^{1}\left(\mathbb{R}^{3}, W\right)$ and

$$
(f(|\cdot|))^{\wedge}(\xi)=\frac{i}{|\xi|} \cdot\left(\operatorname{id}_{\mathbb{R}} \cdot f\right)^{\wedge}(|\xi|)
$$

for all $\xi \in \mathbb{R}^{3} \backslash\{0\}$.

Proof. The function $f(|\cdot|)$ is summable, since

$$
\frac{1}{4 \pi} \int_{\mathbb{R}^{3}}|f(|x|)| d x=\int_{0}^{\infty} r^{2}|f(r)| d r \leq \int_{0}^{1} r|f(r)| d r+\int_{1}^{\infty} r^{2}|f(r)| d r .
$$

Applying the formula

$$
\int_{S_{2}} e^{-i(v \mid \zeta)} d \zeta=\int_{S_{2}} \cos (v \mid \zeta) d \zeta=4 \pi \frac{\sin |v|}{|v|}
$$

where $v \in \mathbb{R}^{3} \backslash\{0\}$ and $d \zeta$ is the surface element on the sphere $S_{2}$, we obtain

$$
\begin{aligned}
(f(|\cdot|))^{\wedge}(\xi) & =(2 \pi)^{-3 / 2} \int_{0}^{\infty} r^{2} \int_{S_{2}} e^{-i r(\xi \mid \zeta)} d \zeta f(r) d r \\
& =\frac{1}{|\xi|}\left(\frac{2}{\pi}\right)^{1 / 2} \int_{0}^{\infty}(\sin r|\xi|) r f(r) d r .
\end{aligned}
$$

The function $r \rightarrow(\sin r|\xi|) r f(r)$ is even and $r \rightarrow(\cos r|\xi|) r f(r)$ is odd. So

$$
\begin{aligned}
\frac{1}{|\xi|}\left(\frac{2}{\pi}\right)^{1 / 2} \int_{0}^{\infty}(\sin r|\xi|) r f(r) d r=\frac{1}{|\xi|}\left(\frac{2}{\pi}\right)^{1 / 2} \frac{1}{2} \int_{\mathbb{R}}(\sin r|\xi|) r f(r) d r \\
=-\frac{i}{|\xi|} \int_{\mathbb{R}} i(\sin r|\xi|) r f(r) \frac{d r}{\sqrt{2 \pi}}=-\frac{i}{|\xi|} \int_{\mathbb{R}} e^{i r|\xi|} r f(r) \frac{d r}{\sqrt{2 \pi}} \\
=\frac{i}{|\xi|} \int_{\mathbb{R}} e^{-i s|\xi|} s f(s) \frac{d s}{\sqrt{2 \pi}}=\frac{i}{|\xi|}\left(\operatorname{id}_{\mathbb{R}} f\right)^{\wedge}(|\xi|),
\end{aligned}
$$

and this is precisely the assertion of the lemma.

(1'.6) Proof of Theorem (1.6). We can choose admissible norms in $W_{1}, W_{2}$ and $W$ such that $\left|v_{1} v_{2}\right| \leq\left|v_{1}\right|\left|v_{2}\right|$ for any $\left(v_{1}, v_{2}\right) \in W_{1} \times W_{2}$. Assuming some regularity conditions, we will easily prove (1.7). We will then reduce the assumptions gradually till we gain Theorem (1.6). 
S tep 1. Assume that

(i) $K=\mathbb{C}$,

(ii) $\left\{w_{1} \neq 0\right\} \cup\left\{w_{2} \neq 0\right\}$ is bounded,

(iii) $w_{1}$ is bounded,

(iv) $\int_{\mathbb{R}}\left|\dot{w}_{2}(r)\right|^{2} d r<\infty$.

It is clear that the functions $f_{1}(r):=-w_{1}(r) / r$ and $f_{2}(r):=\dot{w}_{2}(r) / r$ are even. By $\left(1^{\prime} .4\right)$, we have

$$
\left(f_{k}(|\cdot|)\right)^{\wedge}(\xi)=\frac{i}{|\xi|}\left(\operatorname{id}_{\mathbb{R}} f_{k}\right)^{\wedge}(|\xi|)
$$

for all $\xi \in \mathbb{R}^{3} \backslash\{0\}$ and $k=1,2$. We can rewrite this as

$$
f_{1}(|\cdot|)^{\wedge}(\xi)=-\frac{i}{|\xi|} w_{1}^{\wedge}(|\xi|), \quad f_{2}(|\cdot|)^{\wedge}(\xi)=\frac{i}{|\xi|}\left(\dot{w}_{2}\right)^{\wedge}(|\xi|)=-w_{2}^{\wedge}(|\xi|) .
$$

Hence, by $\left(1^{\prime} .2\right)$, we compute:

$$
\begin{aligned}
\left(f_{1}(|\cdot|) \widetilde{*} f_{2}(|\cdot|)\right)^{\wedge}(\xi) & =f_{1}(|\cdot|)^{\wedge}(\xi) \cdot f_{2}(|\cdot|)^{\wedge}(\xi) \\
& =\frac{i}{|\xi|}\left(w_{1}^{\wedge} w_{2}^{\wedge}\right)(|\xi|)=\frac{i}{|\xi|}\left(w_{1} \widetilde{*} w_{2}\right)^{\wedge}(|\xi|) \\
& =\frac{i}{|\xi|}\left(\operatorname{id}_{\mathbb{R}} f\right)^{\wedge}(|\xi|),
\end{aligned}
$$

where $f(r):=(1 / r)\left(w_{1} \widetilde{*} w_{2}\right)(r)$. Note that $f$ is even and $\int_{0}^{1} r|f(r)| d r<\infty$, $\int_{1}^{\infty} r^{2}|f(r)| d r<\infty$ since $w_{1} * w_{2} \in C_{\mathrm{c}}(\mathbb{R}, W)$. Applying Lemma (1'.3) to $\left(1^{\prime} .7\right)$ we obtain, for all $\xi \in \mathbb{R}^{3} \backslash\{0\}$,

$$
\left(f_{1}(|\cdot|) \widetilde{*} f_{2}(|\cdot|)\right)^{\wedge}(\xi)=(f(|\cdot|))^{\wedge}(\xi) .
$$

Equality of the transforms of summable functions implies equality of these functions almost everywhere, so

$$
f_{1}(|\cdot|) \widetilde{*} f_{2}(|\cdot|)=f(|\cdot|) \quad \text { in } L^{1}\left(\mathbb{R}^{3}, W\right) .
$$

Certainly $f(|\cdot|)$ is continuous on $\mathbb{R}^{3} \backslash\{0\}$. The left-hand side of (1'.8) has the same property, because $f_{k}(|\cdot|) \in L^{2}\left(\mathbb{R}^{3}, W_{k}\right), k=1,2$ (the condition (iv) guarantees this for $k=2$ ). Therefore $\left(f_{1}(|\cdot|) \approx f_{2}(|\cdot|)\right)(x)=f(|x|)$, for $x \in \mathbb{R}^{3} \backslash\{0\}$, i.e.

$$
\left(w_{1} * w_{2}\right)(|x|)=\frac{|x|}{2 \pi}\left(f_{1}(|\cdot|) * f_{2}(|\cdot|)\right)(x) .
$$

The function $w_{1} * w_{2}$ is odd, thus $\left(1^{\prime} .9\right)$ also holds for $x=0$. Finally,

$$
\text { formula (1.7) holds for any } x \in \mathbb{R}^{3} \text {. }
$$

At this moment we interrupt Proof $\left(1^{\prime} .6\right)$. It is continued at $\left(1^{\prime} .16\right)$. 
(1'.11) Lemma (scalar version of Remark (2.1)). If $\kappa:] 0, \infty[\rightarrow[0, \infty]$ is a Borel measurable function, then for all $x \in \mathbb{R}^{3} \backslash\{0\}$,

$$
\left(\kappa(|\cdot|) * \frac{1}{4 \pi|\cdot|}\right)(x)=\frac{1}{|x|} \int_{0}^{|x|} r^{2} \kappa(r) d r+\int_{|x|}^{\infty} r \kappa(r) d r .
$$

Pr o of. We apply Step 1 of Proof ( $1^{\prime}$.6). In view of (L $\nearrow$ ) one can assume that $\kappa$ is a bounded function with bounded support. We choose an even nonnegative function $\psi \in D(\mathbb{R})$ such that $\psi(0)=1$ and $\psi$ is decreasing on $\mathbb{R}_{+}$. Let $\nu \in \mathbb{N} \backslash\{0\}$. The functions

$$
w_{1}(r):=-r \kappa(|r|), \quad w_{2, \nu}(r):=\psi(r / \nu) \cdot|r|
$$

satisfy the conditions (i)-(iv) of Step 1 . As a consequence of ( $\left.1^{\prime} .10\right)$ we get, for all $x \in \mathbb{R}^{3}$,

$$
\left(w_{1} * w_{2, \nu}\right)(|x|)=\frac{|x|}{2 \pi}\left(-\frac{w_{1}(|\cdot|)}{|\cdot|} * \frac{\dot{w}_{2, \nu}(|\cdot|)}{|\cdot|}\right)(x) .
$$

We compute the limit as $\nu \rightarrow \infty$ of the left-hand side of $\left(1^{\prime} .12\right)$ using $(\mathrm{L} /)$ :

$$
\begin{aligned}
\left(w_{1} * w_{2, \nu}\right)(r)= & \int_{\mathbb{R}}(-s) \kappa(|s|) \psi\left(\frac{|r-s|}{\nu}\right)|r-s| d s \\
= & \int_{-\infty}^{0}+\int_{0}^{\infty} \\
= & \int_{0}^{\infty} \tau \kappa(\tau) \psi\left(\frac{|r+\tau|}{\nu}\right)(r+\tau) d \tau \\
& -\int_{0}^{\infty} s \kappa(s) \psi\left(\frac{|r-s|}{\nu}\right)|r-s| d s \\
\rightarrow & \int_{0}^{\infty} \tau \kappa(\tau)(r+\tau) d \tau-\int_{0}^{\infty} s \kappa(s)|r-s| d s \\
= & 2 \int_{0}^{r} \tau^{2} \kappa(\tau) d \tau+2 r \int_{r}^{\infty} \tau \kappa(\tau) d \tau .
\end{aligned}
$$

Next, we compute the limit of the right-hand side of $\left(1^{\prime} .12\right)$ :

$$
\begin{aligned}
\left(\frac{-w_{1}(|\cdot|)}{|\cdot|} * \frac{\dot{w}_{2, \nu}(|\cdot|)}{|\cdot|}\right)(x)= & \frac{1}{\nu}\left(\kappa(|\cdot|) * \psi^{\prime}\left(\frac{|\cdot|}{\nu}\right)\right)(x) \\
& +\left(\kappa(|\cdot|) *\left(\psi\left(\frac{|\cdot|}{\nu}\right) \frac{1}{|\cdot|}\right)\right)(x) \\
= & : \frac{1}{\nu} J_{1}(\nu)+J_{2}(\nu) .
\end{aligned}
$$


The sequence $\left(J_{1}(\nu)\right)_{\nu=1}^{\infty}$ is bounded; from (L $\left./\right)$ it follows that $\lim _{\nu \rightarrow \infty} J_{2}(\nu)=\left(\kappa(|\cdot|) * K_{1}\right)(x)$. Hence for all $x \in \mathbb{R}^{3}$,

$$
\lim _{\nu \rightarrow \infty}\left(-\frac{w_{1}(|\cdot|)}{|\cdot|} * \frac{\dot{w}_{2, \nu}(|\cdot|)}{|\cdot|}\right)(x)=\left(\kappa(|\cdot|) * K_{1}\right)(x) .
$$

We compare the limits of both sides of $\left(1^{\prime} .12\right)$, taking into account $\left(1^{\prime} .13\right)$ and $\left(1^{\prime} .14\right)$.

$\left(1^{\prime} .15\right)$ Corollary. For any $v \in \mathbb{R}^{3}$,

$$
\frac{1}{4 \pi} \int_{S_{2}} \frac{d \zeta}{|\zeta-v|}= \begin{cases}1 & \text { if }|v| \leq 1 \\ 1 /|v| & \text { if }|v| \geq 1\end{cases}
$$

Proof. First, we give a proof based on methods of the classical theory of the Laplace equation. If $|v|>1$, then the function $\left(x \mapsto|x-v|^{-1}\right)$ is harmonic in the ball $\{|x| \leq 1\}$; therefore, by the Gauss theorem,

$$
\frac{1}{4 \pi} \int_{S_{2}} \frac{d \zeta}{|\zeta-v|}=\left.\frac{1}{|x-v|}\right|_{x=0}=\frac{1}{|v|} .
$$

With the aid of (L $\nearrow$ ) one can prove that this formula extends to the case of $|v|=1$ (see the analogous reasoning below). It remains to consider the case of $|v|<1$. Let $0<\Theta<1$. The function

$$
c: \Theta S_{2} \ni v \mapsto \frac{1}{4 \pi} \int_{S_{2}} \frac{d \zeta}{|\zeta-v|} \in \mathbb{R}
$$

is constant, because the Euclidean measure on $S_{2}$ is invariant with respect to any linear isometry of $\mathbb{R}^{3}$. The function

$$
u(v):=\frac{1}{4 \pi} \int_{S_{2}} \frac{d \zeta}{|\zeta-v|}
$$

is a solution of the Dirichlet problem

$$
\Delta u=0 \quad \text { in }\{|x| \leq \Theta\}, \quad c \subset u .
$$

From the maximum principle it follows that $u \equiv$ const $=c$. In particular, $c=u(0)=1$.

In order to show the usefulness of Theorem (1.6) we will present another proof, based just on Lemma $\left(1^{\prime} .11\right)$. One can assume that $v \neq 0$. Fix $\zeta_{0} \in S_{2}$. Let $0<R<\infty$ and put

$$
\left.\kappa_{R}:\right] 0, \infty\left[\ni r \mapsto \left\{\begin{array}{ll}
r^{-2} & \text { for } r \leq R \\
0 & \text { for } r>R
\end{array}\right.\right.
$$


From Lemma (1'.11) it follows that

$$
\begin{aligned}
\left(\kappa_{R}(|\cdot|) * \frac{1}{4 \pi|\cdot|}\right)\left(\zeta_{0}\right) & =\int_{0}^{1} r^{2} \kappa_{R}(r) d r+\int_{1}^{\infty} r \kappa_{R}(r) d r \\
& = \begin{cases}R & \text { for } R \leq 1, \\
1+\ln R & \text { for } R>1 .\end{cases}
\end{aligned}
$$

Thus the function $0<R \mapsto\left(\kappa_{R}(|\cdot|) *(1 /(4 \pi|\cdot|))\right)\left(\zeta_{0}\right)$ is of class $C^{1}$, and moreover,

$$
g(R):=\frac{d}{d R}\left(\kappa_{R}(|\cdot|) * \frac{1}{4 \pi|\cdot|}\right)\left(\zeta_{0}\right)= \begin{cases}1 & \text { for } R \leq 1 \\ 1 / R & \text { for } R>1 .\end{cases}
$$

On the other hand,

$$
\begin{aligned}
\left(\kappa_{R}(|\cdot|) * \frac{1}{4 \pi|\cdot|}\right)\left(\zeta_{0}\right) & =\frac{1}{4 \pi} \int_{|x| \leq R} \frac{d x}{|x|^{2}\left|\zeta_{0}-x\right|} \\
& =\int_{0}^{R} \frac{1}{4 \pi} \int_{S_{2}} \frac{d \zeta}{\left|\zeta_{0}-r \zeta\right|} d r=: \int_{0}^{R} f(r) d r .
\end{aligned}
$$

If $K \subset \mathbb{R}_{+} \backslash\{1\}$ is a compact set, then the function

$$
K \times S_{2} \ni(r, \zeta) \mapsto \frac{1}{\left|\zeta_{0}-r \zeta\right|} \in \mathbb{R}
$$

is bounded, as a continuous function with compact domain. Therefore with the aid of $(\overline{\mathrm{L}})$ it is easy to verify the continuity of $f$ on $\mathbb{R}_{+} \backslash\{1\}$. We know that $\int_{0}^{R} f(r) d r<\infty$ for all $R>0$. If $R_{0} \in \mathbb{R}_{+} \backslash\{1\}$, then $R_{0}$ is a point of continuity of $f$, thus

$$
f\left(R_{0}\right)=\left.\frac{d}{d R} \int_{0}^{R} f(r) d r\right|_{R=R_{0}}=g\left(R_{0}\right) .
$$

We proved that $f=g$ on $\mathbb{R}_{+} \backslash\{1\}$. It remains to compute $f(1)$. The function ] $1, \infty\left[\ni r \mapsto\left|\zeta_{0}-r \zeta\right|\right.$ is increasing, since $(d / d r)\left|\zeta_{0}-r \zeta\right|^{2}>0$ whenever $r>1$. We choose a decreasing sequence $1<r_{\nu} \rightarrow 1$. The sequence $\left(1 /\left|\zeta_{0}-r_{\nu} \zeta\right|\right)$ is increasing, so using ( $\mathrm{L} \nearrow$ ), we compute

$$
g(1) \leftarrow g\left(r_{\nu}\right)=f\left(r_{\nu}\right)=\frac{1}{4 \pi} \int_{S_{2}} \frac{d \zeta}{\left|\zeta_{0}-r_{\nu} \zeta\right|} \rightarrow \frac{1}{4 \pi} \int_{S_{2}} \frac{d \zeta}{\left|\zeta_{0}-\zeta\right|}=f(1) .
$$

So, finally, $f=g$ on $\mathbb{R}_{+}$. It remains to put $\zeta_{0}:=v /|v|$ and to calculate $f(1 /|v|)$. Therefore, Remark (2.1) implies Corollary (1'.15).

$\left(1^{\prime} .15\right)^{*}$ Digression. Conversely, from Corollary $\left(1^{\prime} .15\right)$ one can easily 
derive Remark (2.1). Namely,

$$
\left(\frac{1}{4 \pi|\cdot|} * \kappa(|\cdot|)\right)(x)=\int_{0}^{\infty}\left(\frac{1}{4 \pi} \int_{S_{2}} \frac{d \zeta}{\left|\zeta-r^{-1} x\right|}\right) \cdot r \kappa(r) d r .
$$

( $\left.1^{\prime} .16\right)$ Continuation of Proof ( $\left.1^{\prime} .6\right)$. Step 2. Let us take the additional assumptions (i), (ii), (iii) from Step 1. We choose an approximate identity $\left(h_{\nu}\right)_{\nu=1}^{\infty}$ on $\mathbb{R}$ composed of even test functions. The smooth function $w_{2, \nu}:=h_{\nu} * w_{2}$ is even, $\dot{w}_{2, \nu}\left(=h_{\nu} * \dot{w}_{2}\right)$ has bounded support. According to Step 1 , for all $x \in \mathbb{R}^{3}$,

$$
\left(w_{1} * w_{2, \nu}\right)(|x|)=\frac{|x|}{2 \pi}\left(-\frac{w_{1}(|\cdot|)}{|\cdot|} * \frac{\dot{w}_{2, \nu}(|\cdot|)}{|\cdot|}\right)(x) .
$$

Then $w_{2, \nu}$ tends uniformly to $w_{2}$, since $w_{2}$ is uniformly continuous. From this and $(\overline{\mathrm{L}})$,

$$
w_{1} * w_{2, \nu} \rightarrow w_{1} * w_{2} \text { pointwise } \quad \text { as } \nu \rightarrow \infty .
$$

Next, we compute the limit of the right-hand side of $\left(1^{\prime} .17\right)$ :

$$
\begin{aligned}
\left(\frac{w_{1}(|\cdot|)}{|\cdot|} * \frac{\dot{w}_{2, \nu}(|\cdot|)}{|\cdot|}\right)(x) & =\int_{\mathbb{R}^{3}} \frac{w_{1}(|x-y|)}{|x-y||y|} \cdot \dot{w}_{2, \nu}(|y|) d y \\
& =\int_{0}^{\infty} \int_{S_{2}} \frac{w_{1}(|x-r \zeta|)}{|x / r-\zeta|} d \zeta \cdot \dot{w}_{2, \nu}(r) d r .
\end{aligned}
$$

With the aid of Corollary $\left(1^{\prime} .15\right)$ we estimate

$$
\frac{1}{4 \pi} \int_{S_{2}} \frac{\left|w_{1}(|x-r \zeta|)\right|}{|x / r-\zeta|} d \zeta \leq \begin{cases}\left\|w_{1}\right\|_{L^{\infty}} & \text { if }|x| \leq r \\ \left\|w_{1}\right\|_{L^{\infty}} \cdot(r /|x|) & \text { if }|x| \geq r\end{cases}
$$

In particular, the function

$$
] 0, \infty\left[\ni r \mapsto \int_{S_{2}} \frac{w_{1}(|x-r \zeta|)}{|x / r-\zeta|} d \zeta \in W_{1}\right.
$$

is bounded on the (bounded) subset $\bigcup_{\nu}\left\{\dot{w}_{2, \nu} \neq 0\right\}$. At the same time, $\dot{w}_{2, \nu}=h_{\nu} * \dot{w}_{2} \rightarrow \dot{w}_{2}$ in $L^{1}\left(\mathbb{R}, W_{2}\right)$, since $\int_{\mathbb{R}}\left|\dot{w}_{2}(r)\right| d r<\infty$. Hence

$$
\begin{aligned}
\int_{0}^{\infty} \int_{S_{2}} \frac{w_{1}(|x-r \zeta|)}{|x / r-\zeta|} d \zeta \cdot \dot{w}_{2, \nu}(r) d r & \rightarrow \int_{0}^{\infty} \int_{S_{2}} \frac{w_{1}(|x-r \zeta|)}{|x / r-\zeta|} d \zeta \cdot \dot{w}_{2}(r) d r \\
& =\int_{\mathbb{R}^{3}} \frac{w_{1}(|x-y|)}{|x-y||y|} \cdot \dot{w}_{2}(|y|) d y \\
& =\left(\frac{w_{1}(|\cdot|)}{|\cdot|} * \frac{\dot{w}_{2}(|\cdot|)}{|\cdot|}\right)(x) .
\end{aligned}
$$


Taking this result and $\left(1^{\prime} .18\right)$ into consideration, we compare the limits of both sides of $\left(1^{\prime} .17\right)$ and we obtain $\left(1^{\prime} .10\right)$ by the additional assumptions (i), (ii), (iii).

S te p 3. We additionally assume that

$\left(1^{\prime} .20\right) \quad\left\{w_{1} \neq 0\right\}$ is bounded, $w_{1}$ is bounded and $K=\mathbb{C}$.

We choose $\psi \in D(\mathbb{R})$ such that $\psi(0)=1$ and for $\nu \in \mathbb{N} \backslash\{0\}$ we set $w_{2, \nu}(r):=\psi(|r| / \nu) \cdot w_{2}(r)$. According to Step 2 , for all $x \in \mathbb{R}^{3}$,

$$
\left(w_{1} * w_{2, \nu}\right)(|x|)=\frac{|x|}{2 \pi} \cdot\left(-\frac{w_{1}(|\cdot|)}{|\cdot|} * \frac{\dot{w}_{2, \nu}(|\cdot|)}{|\cdot|}\right)(x) .
$$

From $(\overline{\mathrm{L}})$ it follows that

$$
w_{1} * w_{2, \nu} \rightarrow w_{1} * w_{2} \text { pointwise } \quad \text { as } \nu \rightarrow \infty .
$$

Let $x \in \mathbb{R}^{3}$. Then

$$
\begin{aligned}
\left(\frac{w_{1}(|\cdot|)}{|\cdot|} * \frac{\dot{w}_{2, \nu}(|\cdot|)}{|\cdot|}\right)(x)= & \frac{1}{\nu} \int_{\mathbb{R}^{3}} \frac{w_{1}(|x-y|)}{|x-y|} \cdot \psi^{\prime}\left(\frac{|y|}{\nu}\right) \cdot \frac{w_{2}(|y|)}{|y|} d y \\
& +\int \frac{w_{1}(|x-y|)}{|x-y|} \psi\left(\frac{|y|}{\nu}\right) \cdot \frac{\dot{w}_{2}(|y|)}{|y|} d y \\
= & \frac{1}{\nu} J_{1}(\nu)+J_{2}(\nu) .
\end{aligned}
$$

We estimate

$$
\begin{aligned}
\left|J_{1}(\nu)\right| & \leq \int_{\mathbb{R}^{3}} \frac{\left|w_{1}(|x-y|)\right|}{|x-y|} \cdot\left\|\psi^{\prime}\right\|_{L^{\infty}} \cdot \frac{\left|w_{2}(|y|)\right|}{|y|} d y \\
& =\left\|\psi^{\prime}\right\|_{L^{\infty}} \cdot \int_{0}^{M+|x|} \int_{S_{2}} \frac{\left|w_{1}(|x-r \zeta|)\right|}{|x / r-\zeta|} d \zeta \cdot\left|w_{2}(r)\right| d r
\end{aligned}
$$

where $M:=\sup _{w_{1}(t) \neq 0}|t|$. From this and the inequality $\left(1^{\prime} .19\right)$ it follows that the sequence $\left(J_{1}(\nu)\right)$ is bounded. Also by $\left(1^{\prime} .19\right)$ one can apply $(\overline{\mathrm{L}})$ to the calculation of $\lim _{\nu \rightarrow \infty} J_{2}(\nu)$, namely

$$
\begin{aligned}
J_{2}(\nu) & =\int_{0}^{M+|x|} \psi\left(\frac{r}{\nu}\right) \int_{S_{2}} \frac{w_{1}(|x-r \zeta|)}{|x / r-\zeta|} d \zeta \cdot \dot{w}_{2}(r) d r \\
& \rightarrow \int_{0}^{M+|x|} \int_{S_{2}} \frac{w_{1}(|x-r \zeta|)}{|x / r-\zeta|} d \zeta \cdot \dot{w}_{2}(r) d r \\
& =\left(\frac{w_{1}(|\cdot|)}{|\cdot|} * \frac{\dot{w}_{2}(|\cdot|)}{|\cdot|}\right)(x) .
\end{aligned}
$$


Putting all together gives

$$
\lim _{\nu \rightarrow \infty}\left(\frac{w_{1}(|\cdot|)}{|\cdot|} * \frac{\dot{w}_{2, \nu}(|\cdot|)}{|\cdot|}\right)(x)=\left(\frac{w_{1}(|\cdot|)}{|\cdot|} * \frac{\dot{w}_{2}(|\cdot|)}{|\cdot|}\right)(x) .
$$

Taking this fact and $\left(1^{\prime} .22\right)$ into account, we compare the limits of the both sides of $\left(1^{\prime} .21\right)$ and we get $\left(1^{\prime} .10\right)$ by the additional assumptions $\left(1^{\prime} .20\right)$.

S tep 4 . Now we only assume that $K=\mathbb{C}$. Fix $x_{0} \in \mathbb{R}^{3}$ such that

$$
\left(\left|w_{1}\right| *\left|w_{2}\right|\right)\left(\left|x_{0}\right|\right)<\infty \text { and }\left(\frac{\left|w_{1}(|\cdot|)\right|}{|\cdot|} * \frac{\left|\dot{w}_{2}(|\cdot|)\right|}{|\cdot|}\right)\left(x_{0}\right)<\infty .
$$

By (1.5) there exists a sequence of simple Borel measurable functions $\widetilde{w}_{1, \nu}$ : $\mathbb{R}_{+} \rightarrow W_{1}, \nu=1,2, \ldots$, with bounded supports such that $\left\{\left.\widetilde{w}_{1, \nu} \rightarrow w_{1}\right|_{\mathbb{R}_{+}}\right.$ pointwise as $\nu \rightarrow \infty\}$ and $\left|\widetilde{w}_{1, \nu}(r)\right| \leq\left|w_{1}(r)\right|$ for all $r \in \mathbb{R}_{+}$and all $\nu$. For every $\nu$ the function

$$
w_{1, \nu}: \mathbb{R} \ni r \mapsto \begin{cases}\widetilde{w}_{1, \nu}(r) & \text { if } r>0 \\ -\widetilde{w}_{1, \nu}(-r) & \text { if } r<0\end{cases}
$$

is Borel measurable, simple, odd and has bounded support. Moreover, $w_{1, \nu}$ $\rightarrow w_{1}$ pointwise as $\nu \rightarrow \infty$ and $\left|w_{1, \nu}(r)\right| \leq\left|w_{1}(r)\right|$ for all $r \in \mathbb{R}$ and all $\nu$. According to Step 3, for all $\nu \in \mathbb{N}$,

$$
\left(w_{1, \nu} * w_{2}\right)\left(\left|x_{0}\right|\right)=\frac{\left|x_{0}\right|}{2 \pi}\left(-\frac{w_{1, \nu}(|\cdot|)}{|\cdot|} * \frac{\dot{w}_{2}(|\cdot|)}{|\cdot|}\right)\left(x_{0}\right) .
$$

Applying $(\overline{\mathrm{L}})$ we pass to the limit on both sides of $\left(1^{\prime} .23\right)$ and we get

$$
\left(w_{1} * w_{2}\right)\left(\left|x_{0}\right|\right)=\frac{\left|x_{0}\right|}{2 \pi}\left(-\frac{w_{1}(|\cdot|)}{|\cdot|} * \frac{\dot{w}_{2}(|\cdot|)}{|\cdot|}\right)\left(x_{0}\right) .
$$

Step 5. It remains to deal with the case of $K=\mathbb{R}$. We recall the complexification method. $W^{2}$ is a complex linear space with the following multiplication of a scalar and a vector:

$$
\alpha \cdot\left(v^{\prime}, v^{\prime \prime}\right):=\left(\alpha_{1} v^{\prime}-\alpha_{2} v^{\prime \prime}, \alpha_{1} v^{\prime \prime}+\alpha_{2} v^{\prime}\right),
$$

where $\alpha \in \mathbb{C}, \alpha_{1}:=\operatorname{Re} \alpha$ and $\alpha_{2}:=\operatorname{Im} \alpha$. The locally convex space $W^{2}$ (=W $\times W$ with the product topology) has a bound7ed neighbourhood of zero, therefore it is a $\mathbb{C}$-normed space. One can choose an admissible $\mathbb{C}$-norm in $W^{2}$ such that

$$
\forall\left(v^{\prime}, v^{\prime \prime}\right) \in W^{2}:\left|\left(v^{\prime}, v^{\prime \prime}\right)\right| \leq\left|v^{\prime}\right|+\left|v^{\prime \prime}\right| .
$$

Likewise we fit $\mathbb{C}$-norms in $W_{1}^{2}, W_{2}^{2}$ (corresponding to the topologies). The map

$\left(1^{\prime} .25\right) W_{1}^{2} \times W_{2}^{2} \ni\left(\left(v_{1}^{\prime}, v_{1}^{\prime \prime}\right),\left(v_{2}^{\prime}, v_{2}^{\prime \prime}\right)\right) \mapsto\left(v_{1}^{\prime} v_{2}^{\prime}-v_{1}^{\prime \prime} v_{2}^{\prime \prime}, v_{1}^{\prime} v_{2}^{\prime \prime}+v_{1}^{\prime \prime} v_{2}^{\prime}\right) \in W^{2}$ is continuous and $\mathbb{C}$-bilinear. Consider the canonical injection $l: W \ni v \mapsto$ $(v, 0) \in W^{2}$ and the analogously defined injections $l_{1}: W_{1} \rightarrow W_{1}^{2}, l_{2}: W_{2} \rightarrow$ 
$W_{2}^{2}$. It is clear that $l_{1} \circ w_{1}$ is odd and Borel measurable, $l_{2} \circ w_{2}$ is even and absolutely continuous, $\left(l_{2} \circ w_{2}\right)^{\prime}=l_{2} \circ \dot{w}_{2}$. Fix $x_{0} \in \mathbb{R}^{3}$ such that

$$
\left(\left|w_{1}\right| *\left|w_{2}\right|\right)\left(\left|x_{0}\right|\right)<\infty \quad \text { and } \quad\left(\frac{\left|w_{1}(|\cdot|)\right|}{|\cdot|} * \frac{\left|\dot{w}_{2}(|\cdot|)\right|}{|\cdot|}\right)\left(x_{0}\right)<\infty .
$$

Then

$$
\begin{array}{r}
\left(\left|l_{1} \circ w_{1}\right| *\left|l_{2} \circ w_{2}\right|\right)\left(\left|x_{0}\right|\right)<\infty \text { and } \\
\left(\frac{\left|\left(l_{1} \circ w_{1}\right)(|\cdot|)\right|}{|\cdot|} * \frac{\left|\left(l_{2} \circ w_{2}\right)^{\prime}(|\cdot|)\right|}{|\cdot|}\right)\left(x_{0}\right)<\infty
\end{array}
$$

and by Step 4,

$\left(1^{\prime} .26\right) \quad\left(\left(l_{1} \circ w_{1}\right) *\left(l_{2} \circ w_{2}\right)\right)\left(\left|x_{0}\right|\right)$

$$
=\frac{\left|x_{0}\right|}{2 \pi}\left(-\frac{\left(l_{1} \circ w_{1}\right)(|\cdot|)}{|\cdot|} * \frac{\left(l_{2} \circ w_{2}\right)^{\prime}(|\cdot|)}{|\cdot|}\right)\left(x_{0}\right)
$$

where the convolutions on both sides of $\left(1^{\prime} .26\right)$ are defined with the aid of the "multiplication" $\left(1^{\prime} .25\right)$. We rearrange the equality $\left(1^{\prime} .26\right)$, using the identity $l_{1}\left(v_{1}\right) l_{2}\left(v_{2}\right)=l\left(v_{1} v_{2}\right)$ and linearity of the integral to obtain

$$
l\left(\left(w_{1} * w_{2}\right)\left(\left|x_{0}\right|\right)\right)=l\left(\frac{\left|x_{0}\right|}{2 \pi} \cdot\left(-\frac{w_{1}(|\cdot|)}{|\cdot|} * \frac{\dot{w}_{2}(|\cdot|)}{|\cdot|}\right)\left(x_{0}\right)\right),
$$

which proves $\left(1^{\prime} .24\right)$. The proof of Theorem (1.6) is complete.

\section{$2^{\prime}$. Proofs}

$\left(2^{\prime} .1\right)$ Proof of Remark (2.1). See Lemma (1'.11) (and reduction to the scalar version in $\left.\left(2^{\prime} .5\right)\right)$ or Digression $\left(1^{\prime} .15\right)^{*}$.

$\left(2^{\prime} .2\right)$ Proof of $\operatorname{Remark}(2.3)$. By $(\mathrm{L} \nearrow)$ one can assume that $\kappa$ is bounded and has bounded support. In Theorem (1.6) we substitute

$$
w_{1}(r)=-r \kappa(|r|), \quad w_{2}(r)=-\frac{1}{c} e^{-(c / 2) r^{2}} .
$$

$\left(2^{\prime} .3\right)$ Lemma (scalar version of Lemma (2.4)). If $\left.\kappa:\right] 0, \infty[\rightarrow[0, \infty]$ is a Borel measurable function, then for all $x \in \mathbb{R}^{3} \backslash\{0\}$,

$$
\frac{1}{\pi}\left(\frac{1}{a+|\cdot|^{2}} * \kappa(|\cdot|)\right)(x)=\frac{1}{|x|} \int_{0}^{\infty} r\left(\ln \frac{a+(r+|x|)^{2}}{a+(r-|x|)^{2}}\right) \kappa(r) d r .
$$

Proof. In view of (L $\nearrow$ ) one can assume that $\kappa$ is a bounded function with bounded support. We put $w_{1}(r)=-r \kappa(|r|), w_{2}(r)=\frac{1}{2} \ln \left(a+r^{2}\right)$ and apply Theorem (1.6).

$\left(2^{\prime} .5\right)$ Pro of of Lemma (2.4). By (1.5) one can assume the separa- 
bility of $W$. The left-hand side of (2.5) exists, since

$$
\left(\frac{1}{a+|\cdot|^{2}} *|\kappa(|\cdot|)|\right)(x)=\int_{0}^{\infty} \int_{S_{2}} \frac{r^{2} d \zeta}{a+\mid x-r \zeta^{2}} \cdot|\kappa(r)| d r<\infty .
$$

The right-hand side of (2.5) also exists, because by $\left(2^{\prime} .6\right)$ and Lemma $\left(2^{\prime} .3\right)$,

$$
\int_{0}^{\infty} r\left(\ln \frac{a+(r+|x|)^{2}}{a+(r-|x|)^{2}}\right)|\kappa(r)| d r=\frac{|x|}{\pi}\left(\frac{1}{a+|\cdot|^{2}} *|\kappa(|\cdot|)|\right)(x)<\infty .
$$

Suppose that Lemma (2.4) is true for $W=\mathbb{R}$. Then in the general case, for all $l \in L(W, \mathbb{R})$,

$$
\begin{aligned}
l\left(\frac{1}{\pi}\left(\frac{1}{a+|\cdot|^{2}} * \kappa(|\cdot|)\right)(x)\right) & =\frac{1}{\pi}\left(\frac{1}{a+|\cdot|^{2}} *(l \circ \kappa)(|\cdot|)\right)(x) \\
& =\frac{1}{|x|} \int_{0}^{\infty} r\left(\ln \frac{a+(r+|x|)^{2}}{a+(r-|x|)^{2}}\right)(l \circ \kappa)(r) d r \\
& =l\left(\frac{1}{|x|} \int_{0}^{\infty} r\left(\ln \frac{a+(r+|x|)^{2}}{a+(r-|x|)^{2}}\right) \kappa(r) d r\right)
\end{aligned}
$$

and hence

$$
\frac{1}{\pi}\left(\frac{1}{a+|\cdot|^{2}} * \kappa(|\cdot|)\right)(x)=\frac{1}{|x|} \int_{0}^{\infty} r\left(\ln \frac{a+(r+|x|)^{2}}{a+(r-|x|)^{2}}\right) \kappa(r) d r
$$

because, by the separability of $W$ and by the Hahn-Banach theorem, the linear continuous real functions separate points in $W$. Therefore one can take $W=\mathbb{R}$, and apply Lemma $\left(2^{\prime} .3\right)$ to the functions $\kappa^{+}:=\max \{0, \kappa\}$, $\kappa^{-}:=\max \{0,-\kappa\}$.

$\left(2^{\prime} .8\right)$ Lemma (scalar version of Corollary (2.6)). If $\left.\kappa:\right] 0, \infty[\rightarrow[0, \infty]$ is a Borel measurable function, then for all $x \in \mathbb{R}^{3}$,

$\left(2^{\prime} .9\right) \frac{1}{4 \pi}\left(\frac{1}{\left(a+|\cdot|^{2}\right)^{2}} * \kappa(|\cdot|)\right)(x)=\int_{0}^{\infty} \frac{r^{2} \kappa(r) d r}{\left(a+(r-|x|)^{2}\right)\left(a+(r+|x|)^{2}\right)}$.

Proof. In view of (L $\nearrow$ ) one can assume that $\kappa$ is a bounded function with bounded support. It is easy to verify $\left(2^{\prime} .9\right)$ for $x=0$, and for $x \neq 0$ it may be immediately obtained by differentiation of $\left(2^{\prime} .4\right)$ with respect to $a$. It is worth while, however, to investigate the proof of $\left(2^{\prime} .9\right)$ based directly on Theorem (1.6). Namely, for $w_{1}(r)=-r \kappa(|r|), w_{2}(r)=-1 /\left(2\left(a+r^{2}\right)\right)$ the formula (1.7) takes the form

$$
\left(\kappa(|\cdot|) * \frac{1}{\left(a+|\cdot|^{2}\right)^{2}}\right)(x)=\frac{\pi}{|x|} \int_{\mathbb{R}} \frac{r \kappa(|r|) d r}{a+(r-|x|)^{2}},
$$

which, after a change of variables, is equivalent to $\left(2^{\prime} .9\right)$. 
$\left(2^{\prime} .11\right)$ Proof of Corollary (2.6). In view of Lemma $\left(2^{\prime} .8\right)$, it is a slight modification of the argument $\left(2^{\prime} .5\right)$.

$\left(2^{\prime} .12\right)$ Proof of (2.10). Both sides of (2.10) are continuous on $\mathbb{R}^{3}$, thus it suffices to show their equality on $\mathbb{R}^{3} \backslash\{0\}$. From $\left(2^{\prime} .10\right)$ it follows that for all $x \in \mathbb{R}^{3} \backslash\{0\}$,

$$
\begin{array}{r}
\left(\frac{1}{a^{2}+|\cdot|^{2}} * \frac{1}{\left(b^{2}+|\cdot|^{2}\right)^{2}}\right)(x)=\frac{\pi}{|x|} \int_{\mathbb{R}} \frac{r d r}{\left(a^{2}+r^{2}\right)\left(b^{2}+(r-|x|)^{2}\right)} \\
\quad=\frac{\pi}{|x|} \lim _{R \rightarrow \infty}\left(\int_{-R}^{R} f(z) d z+\int_{\Gamma_{R}} f(z) d z-\int_{\Gamma_{R}} f(z) d z\right),
\end{array}
$$

where $\Gamma_{R}:[0, \pi] \ni \varphi \mapsto R e^{i \varphi} \in \mathbb{C}$ and

$$
\begin{aligned}
f(z) & :=\frac{z}{\left(a^{2}+z^{2}\right)\left(b^{2}+(z-|x|)^{2}\right)} \\
& =\frac{z}{(z-i a)(z+i a)(z-|x|-i b)(z-|x|+i b)} .
\end{aligned}
$$

For sufficiently large $R$ we have

$$
\int_{-R}^{R} f(z) d z+\int_{\Gamma_{R}} f(z) d z=2 \pi i\left(\operatorname{res}_{i a} f+\operatorname{res}_{|x|+i b} f\right),
$$

where

$$
\begin{aligned}
\operatorname{res}_{i a} f & =\left.\frac{z}{(z+i a)(z-|x|-i b)(z-|x|+i b)}\right|_{z=i a} \\
& =\frac{1}{2(i(a-b)-|x|)(i(a+b)-|x|)}, \\
\operatorname{res}_{|x|+i b} f & =\left.\frac{z}{(z-i a)(z+i a)(z-|x|+i b)}\right|_{z=|x|+i b} \\
& =-\frac{|x|+i b}{2 i b(i(a-b)-|x|)(i(a+b)+|x|)} .
\end{aligned}
$$

After a rearrangement we obtain

$$
\int_{-R}^{R} f(z) d z+\int_{\Gamma_{R}} f(z) d z=\frac{\pi|x|}{b} \cdot \frac{1}{(a+b)^{2}+|x|^{2}} .
$$

The formulas $\left(2^{\prime} .13\right),\left(2^{\prime} .14\right)$ imply $(2.10)$, since

$$
\lim _{R \rightarrow \infty} \int_{\Gamma_{R}} f(z) d z=0
$$


$\left(2^{\prime} .15\right)$ P r o of of (2.9). Let $x \in \mathbb{R}^{3} \backslash\{0\}$. Applying (2.10) we compute:

$$
\begin{aligned}
\frac{d}{d b}\left(\frac{1}{a^{2}+|\cdot|^{2}} * \frac{1}{b^{2}+|\cdot|^{2}}\right)(x) & =-2 b\left(\frac{1}{a^{2}+|\cdot|^{2}} * \frac{1}{\left(b^{2}+|\cdot|^{2}\right)^{2}}\right)(x) \\
& =-\frac{2 \pi^{2}}{(a+b)^{2}+|x|^{2}} \\
& =\frac{d}{d b}\left(-\frac{2 \pi^{2}}{|x|} \arctan \frac{a+b}{|x|}\right) .
\end{aligned}
$$

Consequently, there exists $C \in \mathbb{R}$ such that for all $b>0$,

$$
\left(\frac{1}{a^{2}+|\cdot|^{2}} * \frac{1}{b^{2}+|\cdot|^{2}}\right)(x)=C-\frac{2 \pi^{2}}{|x|} \arctan \frac{a+b}{|x|} .
$$

Passing to the limit on both sides of $\left(2^{\prime} .16\right)$ as $b \rightarrow \infty$, we get

$$
C=\frac{2 \pi^{2}}{|x|} \cdot \frac{\pi}{2} \text {. }
$$

It remains to apply the formula $\forall r>0: \arctan r+\arctan (1 / r)=\pi / 2$.

$\left(2^{\prime} .17\right)$ Proof of (2.11). We differentiate both sides of (2.10) with respect to $a$.

$\left(2^{\prime} .18\right)$ Pro of of (2.12). We write (2.11) in the form

$$
\frac{1}{\left(a^{2}+|\cdot|^{2}\right)^{2}} * \frac{1}{\left(b^{2}+|\cdot|^{2}\right)^{2}}=\frac{\pi^{2}}{b} \cdot \frac{a+b}{a} \cdot \frac{1}{\left((a+b)^{2}+|\cdot|^{2}\right)^{2}}
$$

and differentiate it with respect to $a$. After a rearrangement we obtain $\left(2^{\prime} .19\right) \frac{1}{\left(a^{2}+|\cdot|^{2}\right)^{3}} * \frac{1}{\left(b^{2}+|\cdot|^{2}\right)^{2}}$

$$
=\frac{\pi^{2}}{a^{2}} \frac{1}{\left((a+b)^{2}+|\cdot|^{2}\right)^{2}}\left(\frac{1}{4 a}+\frac{(a+b)^{2}}{b} \frac{1}{(a+b)^{2}+|\cdot|^{2}}\right) .
$$

We differentiate both sides of $\left(2^{\prime} .19\right)$ again with respect to $a$.

$\left(2^{\prime} .20\right)$ Pro of of (2.13). In Theorem (1.6) we substitute $\left\{w_{1}(r)=-r \kappa(|r|), w_{2}(r)=\frac{1}{2}\left(r^{2}-\varrho^{2}\right) \cdot \chi_{\varrho}(r)\right\} \quad$ or, in the other case, $\left\{w_{1}(r)=-r \kappa(|r|), w_{2}(r)=\frac{1}{3}\left(|r|^{3}-\varrho^{3}\right) \cdot \chi_{\varrho}(r)\right\}$.

$\left(2^{\prime} .21\right)$ Proof of Remark (2.14). In Theorem (1.6) we substitute $w_{1}(r)=-r \kappa(|r|), \quad w_{2}(r)=\frac{1}{2-\lambda}\left(\varrho^{2-\lambda} \chi_{\varrho}(r)+|r|^{2-\lambda}\left(1-\chi_{\varrho}(r)\right)\right)$. 


\section{$3^{\prime}$. Proofs}

$\left(3^{\prime} .1\right)$ Proof of Remark (3.1). First, consider the case of $\gamma \leq 0$. Using the triangle inequality $|y| \leq|x|+|y-x|$, we estimate

$$
\begin{aligned}
\left((1+|\cdot|)^{-\gamma} * f\right)(x) & =\int_{|x-y| \leq \varrho}(1+|y|)^{|\gamma|} f(x-y) d y \\
& \leq(1+|x|+\varrho)^{|\gamma|} \int_{|x-y| \leq \varrho} f(x-y) d y \\
& =(1+|x|+\varrho)^{|\gamma|} \cdot\|f\|_{L^{1}},
\end{aligned}
$$

i.e.

$$
(1+|x|)^{\gamma}\left((1+|\cdot|)^{-\gamma} * f\right)(x) \leq\left(\frac{1+|x|+\varrho}{1+|x|}\right)^{|\gamma|} \cdot\|f\|_{L^{1}} .
$$

The right-hand side of $\left(3^{\prime} .2\right)$ is majorized by the right-hand side of (3.2), since

$$
\frac{d}{d r} \frac{1+r+\varrho}{1+r} \leq 0 \quad \text { on } \mathbb{R}_{+} .
$$

It remains to consider the case of $\gamma>0$. If $|x| \leq \varrho$, then at once $(1+|x|)^{\gamma}((1+$ $\left.|\cdot|)^{-\gamma} * f\right)(x) \leq(1+\varrho)^{\gamma}\|f\|_{L^{1}}$, because $(1+|\cdot|)^{-\gamma} * f \leq 1 * f \equiv\|f\|_{L^{1}}$. Assume that $|x|>\varrho$. Again with the aid of the triangle inequality we estimate

$$
\begin{aligned}
\left((1+|\cdot|)^{-\gamma} * f\right)(x) & =\int_{|x-y| \leq \varrho}(1+|y|)^{-\gamma} f(x-y) d y \\
& \leq(1+|x|-\varrho)^{-\gamma} \int_{|x-y| \leq \varrho} f(x-y) d y \\
& =(1+|x|-\varrho)^{-\gamma}\|f\|_{L^{1}},
\end{aligned}
$$

that is,

$$
(1+|x|)^{\gamma}\left((1+|\cdot|)^{-\gamma} * f\right)(x) \leq\left(\frac{1+|x|}{1+|x|-\varrho}\right)^{\gamma} \cdot\|f\|_{L^{1}} .
$$

The right-hand side of $\left(3^{\prime} .3\right)$ is majorized by the right-hand side of (3.2), since

$$
\frac{d}{d r} \frac{1+r}{1+r-\varrho} \leq 0 \quad \text { in }[\varrho, \infty[.
$$

$\left(3^{\prime} .4\right)$ Proof of Lemma (3.3). Define

$$
M:=\sup _{x \in \mathbb{R}^{3}}(1+|x|)^{\lambda}\left((1+|\cdot|)^{-\lambda} *(1+|\cdot|)^{-\lambda}\right)(x)
$$

and let $x_{0} \in \mathbb{R}^{3}$. Then 


$$
\begin{aligned}
\left(1+\left|x_{0}\right|\right)^{\gamma}\left((1+|\cdot|)^{-\gamma} *(1+|\cdot|)^{-\lambda}\right)\left(x_{0}\right) & \\
= & \int_{|x| \leq\left|x_{0}\right|}\left(\frac{1+\left|x_{0}\right|}{1+|x|}\right)^{\gamma}\left(1+\left|x_{0}-x\right|\right)^{-\lambda} d x \\
& \quad+\int_{|x|>\left|x_{0}\right|}\left(\frac{1+\left|x_{0}\right|}{1+|x|}\right)^{\gamma}\left(1+\left|x_{0}-x\right|\right)^{-\lambda} d x \\
\leq & \int_{|x| \leq\left|x_{0}\right|}\left(\frac{1+\left|x_{0}\right|}{1+|x|}\right)^{\lambda}\left(1+\left|x_{0}-x\right|\right)^{-\lambda} d x \\
& \quad+\int_{|x|>\left|x_{0}\right|}\left(1+\left|x_{0}-x\right|\right)^{-\lambda} d x \leq M+\int_{\mathbb{R}^{3}}(1+|y|)^{-\lambda} d y .
\end{aligned}
$$

Therefore for every $\gamma \in[0, \lambda]$ the left-hand side of (3.4) is majorized by $M+\|H\|_{L^{1}}$, where $H:=(1+|\cdot|)^{-\lambda}$. Thus it suffices to prove that $M<\infty$. Let $H_{0}:=\chi_{1}(|\cdot|) \cdot H, H_{\infty}:=H-H_{0}$. Then

$\left(3^{\prime} .5\right) \quad H * H=\left(H_{0} * H\right)+\left(H_{\infty} * H_{0}\right)+\left(H_{\infty} * H_{\infty}\right) \leq 2\left(H * H_{0}\right)+f(|\cdot|) * f(|\cdot|)$,

where $f$ stands for the function (2.15) for $\varrho=1$. By Remark (3.1),

$$
\sup _{x \in \mathbb{R}^{3}}(1+|x|)^{\gamma}\left(H * H_{0}\right)(x) \leq 2^{\lambda}\|H\|_{L^{1}},
$$

therefore, taking into account $\left(3^{\prime} .5\right)$, it suffices to show that

$$
\sup _{x \in \mathbb{R}^{3}}(1+|x|)^{\lambda}(f(|\cdot|) * f(|\cdot|))(x)<\infty .
$$

If $f(|\cdot|) \in L^{2}\left(\mathbb{R}^{3}\right)$ then $f(|\cdot|) * f(|\cdot|) \in L^{\infty}\left(\mathbb{R}^{3}\right)$. Thus we only need to prove that

$$
\varlimsup_{x \rightarrow \infty}|x|^{\lambda}(f(|\cdot|) * f(|\cdot|))(x)<\infty .
$$

Let $x \in \mathbb{R}^{3},|x|>2$. We substitute $\kappa=f$ in (2.16) and estimate

$$
\begin{aligned}
\frac{\lambda-2}{2 \pi}(f(|\cdot|) & * f(|\cdot|))(x) \\
& =\frac{1}{|x|} \int_{|x|-1}^{|x|+1} r^{1-\lambda} d r+\frac{1}{|x|}\left(\int_{-\infty}^{|x|-1}+\int_{|x|+1}^{\infty}\right)|r-| x||^{2-\lambda} r f(|r|) d r \\
& \leq \frac{1}{|x|} \int_{|x|-1}^{|x|+1}\left(\frac{|x|}{2}\right)^{1-\lambda} d r+\frac{1}{|x|}\left(\int_{-\infty}^{0}+\int_{0}^{|x|-1}+\int_{|x|+1}^{\infty}\right)(\ldots) d r
\end{aligned}
$$




$$
\begin{aligned}
& =\left(\frac{2}{|x|}\right)^{\lambda}+\frac{1}{|x|}\left(-\int_{1}^{\infty} r^{1-\lambda}(r+|x|)^{2-\lambda} d r\right. \\
& \left.+\int_{1}^{|x|-1} r^{1-\lambda}(|x|-r)^{2-\lambda} d r+\int_{|x|+1}^{\infty} r^{1-\lambda}(r-|x|)^{2-\lambda} d r\right) \\
& \leq\left(\frac{2}{|x|}\right)^{\lambda}+\frac{1}{|x|}\left(-\int_{1}^{|x| / 2} r^{1-\lambda}(r+|x|)^{2-\lambda} d r\right. \\
& \left.+\int_{1}^{|x|-1} r^{1-\lambda}(|x|-r)^{2-\lambda} d r+\int_{|x|+1}^{\infty}|x|^{1-\lambda}(r-|x|)^{2-\lambda} d r\right) \\
& =\left(\frac{2}{|x|}\right)^{\lambda}+\frac{1}{|x|}\left((\lambda-2) \int_{1}^{|x| / 2} r^{1-\lambda} \int_{|x|-r}^{|x|+r} t^{1-\lambda} d t d r\right. \\
& \left.+\int_{|x| / 2}^{|x|-1} r^{1-\lambda}(|x|-r)^{2-\lambda} d r+|x|^{1-\lambda} \int_{|x|+1}^{\infty}(r-|x|)^{2-\lambda} d r\right) \\
& \leq\left(\frac{2}{|x|}\right)^{\lambda}+\frac{1}{|x|}\left((\lambda-2) \int_{1}^{|x| / 2} r^{1-\lambda} \int_{|x|-r}^{|x|+r}\left(\frac{|x|}{2}\right)^{1-\lambda} d t d r\right. \\
& \left.+\int_{|x| / 2}^{|x|-1}\left(\frac{|x|}{2}\right)^{1-\lambda}(|x|-r)^{2-\lambda} d r+|x|^{1-\lambda} \int_{1}^{\infty} s^{2-\lambda} d s\right) \\
& =\left(\frac{2}{|x|}\right)^{\lambda}+(\lambda-2)\left(\frac{2}{|x|}\right)^{\lambda} \int_{1}^{|x| / 2} r^{2-\lambda} d r+\frac{1}{2}\left(\frac{2}{|x|}\right)^{\lambda} \int_{1}^{|x| / 2} s^{2-\lambda} d s+\frac{|x|^{-\lambda}}{\lambda-3} \\
& \leq\left(\frac{2}{|x|}\right)^{\lambda}+(\lambda-2)\left(\frac{2}{|x|}\right)^{\lambda} \int_{1}^{\infty} r^{2-\lambda} d r+\frac{1}{2}\left(\frac{2}{|x|}\right)^{\lambda} \int_{1}^{\infty} s^{2-\lambda} d s+\frac{|x|^{-\lambda}}{\lambda-3} \\
& =|x|^{-\lambda}\left(2^{\lambda}+\frac{\lambda-2}{\lambda-3} \cdot 2^{\lambda}+\frac{2^{\lambda-1}}{\lambda-3}+\frac{1}{\lambda-3}\right)=:|x|^{-\lambda} \cdot R .
\end{aligned}
$$

Hence

$$
\frac{\lambda-2}{2 \pi} \sup _{|x|>2}|x|^{\lambda}(f(|\cdot|) * f(|\cdot|))(x) \leq R,
$$

which, in particular, implies $\left(3^{\prime} .7\right)$.

$\left(3^{\prime} .8\right)$ Proof of Theorem (3.5). Let $\left(x_{\nu}\right) \in\left(\mathbb{R}^{3}\right)^{\mathbb{N}}$ with $x_{\nu} \rightarrow \infty$ 
(i.e. $\left|x_{\nu}\right| \rightarrow \infty$ as $\nu \rightarrow \infty$ ). We decompose

$$
\begin{aligned}
\left|x_{\nu}\right|^{\gamma}(\varphi * w) & \left(x_{\nu}\right)-\int_{\mathbb{R}^{3}} \varphi(y) d y \cdot w_{\infty}\left(\frac{x_{\nu}}{\left|x_{\nu}\right|}\right) \\
= & \int_{\mathbb{R}^{3}} \varphi(y)\left(\left|x_{\nu}\right|^{\gamma} w\left(x_{\nu}-y\right)-w_{\infty}\left(\frac{x_{\nu}}{\left|x_{\nu}\right|}\right)\right) d y \\
= & \int_{\mathbb{R}^{3}} \varphi(y)\left(\left(\frac{\left|x_{\nu}\right|}{1+\left|x_{\nu}-y\right|}\right)^{\gamma}-1\right)\left(1+\left|x_{\nu}-y\right|\right)^{\gamma} w\left(x_{\nu}-y\right) d y \\
& +\int_{\mathbb{R}^{3}} \varphi(y)\left(\left(1+\left|x_{\nu}-y\right|\right)^{\gamma}-\left|x_{\nu}-y\right|^{\gamma}\right) w\left(x_{\nu}-y\right) d y \\
& +\int_{\mathbb{R}^{3} \backslash\left\{x_{\nu}\right\}} \varphi(y)\left(\left|x_{\nu}-y\right|^{\gamma} w\left(x_{\nu}-y\right)-w_{\infty}\left(\frac{x_{\nu}-y}{\left|x_{\nu}-y\right|}\right)\right) d y \\
& +\int_{\mathbb{R}^{3} \backslash\left\{x_{\nu}\right\}} \varphi(y)\left(w_{\infty}\left(\frac{x_{\nu}-y}{\left|x_{\nu}-y\right|}\right)-w_{\infty}\left(\frac{x_{\nu}}{\left|x_{\nu}\right|}\right)\right) d y \\
=: & I_{1}(\nu)+I_{2}(\nu)+I_{3}(\nu)+I_{4}(\nu) .
\end{aligned}
$$

We will prove that for all $k \in\{1,2,3,4\}$,

$$
\lim _{\nu \rightarrow \infty} I_{k}(\nu)=0 .
$$

In view of Lemma (3.3), for $\Gamma>3$ and $0 \leq \alpha \leq \Gamma$,

$$
C_{\Gamma}(\alpha):=\sup _{x \in \mathbb{R}^{3}}(1+|x|)^{\alpha}\left((1+|\cdot|)^{-\alpha} *(1+|\cdot|)^{-\Gamma}\right)(x)<\infty .
$$

(The letter $\Gamma$ in the present proof has nothing to do with the Euler function from Example (1.3).) We choose admissible norms in $W_{1}, W_{2}, W$ such that $\left|v_{1} v_{2}\right| \leq\left|v_{1}\right| \cdot\left|v_{2}\right|$ for $\left(v_{1}, v_{2}\right) \in W_{1} \times W_{2}$. The assumption (3.6) yields

$$
C:=\sup _{x \in \mathbb{R}^{3}}\left\{(1+|x|)^{\lambda}|\varphi(x)|,(1+|x|)^{\gamma}|w(x)|\right\}<\infty .
$$

First, we will show that $\left(3^{\prime} .9\right)$ holds for $k \in\{2,3,4\}$. After the rearrangement

$$
I_{2}(\nu)=\int_{\mathbb{R}^{3}} \varphi(y) \int_{\left|x_{\nu}-y\right|}^{1+\left|x_{\nu}-y\right|} \gamma t^{\gamma-1} d t w\left(x_{\nu}-y\right) d y,
$$

we estimate

$$
\left|I_{2}(\nu)\right| \leq \gamma C^{2} \int_{\mathbb{R}^{3}}(1+|y|)^{-\lambda} \int_{\left|x_{\nu}-y\right|}^{1+\left|x_{\nu}-y\right|} t^{\gamma-1} d t\left(1+\left|x_{\nu}-y\right|\right)^{-\gamma} d y .
$$


If $\gamma \geq 1$, then $\int_{\left|x_{\nu}-y\right|}^{1+\left|x_{\nu}-y\right|} t^{\gamma-1} d t \leq\left(1+\left|x_{\nu}-y\right|\right)^{\gamma-1}$ and hence

$\left|I_{2}(\nu)\right| \leq \gamma C^{2}\left((1+|\cdot|)^{-\lambda} *(1+|\cdot|)^{-1}\right)\left(x_{\nu}\right) \leq \gamma C^{2}\left(1+\left|x_{\nu}\right|\right)^{-1} \cdot C_{\lambda}(1) \rightarrow 0$

as $\nu \rightarrow \infty$. If $\gamma<1$ and $y \neq x_{\nu}$, then

$$
\int_{\left|x_{\nu}-y\right|}^{1+\left|x_{\nu}-y\right|} t^{\gamma-1} d t \leq\left|x_{\nu}-y\right|^{\gamma-1} \leq\left(1+\left|x_{\nu}-y\right|\right)^{\gamma}\left|x_{\nu}-y\right|^{-1}
$$

and by Remark (2.1),

$$
\begin{aligned}
\left|I_{2}\left(x_{\nu}\right)\right| & \leq \gamma C^{2} \int_{\mathbb{R}^{3}}(1+|y|)^{-\lambda}\left|x_{\nu}-y\right|^{-1} d y \\
& =4 \pi \gamma C^{2}\left(\frac{1}{4 \pi|\cdot|} *(1+|\cdot|)^{-\lambda}\right)\left(x_{\nu}\right) \\
& \leq 4 \pi \gamma C^{2}\left(\frac{1}{\left|x_{\nu}\right|} \int_{0}^{\infty} \frac{r^{2} d r}{(1+r)^{\lambda}}+\int_{\left|x_{\nu}\right|}^{\infty} \frac{r d r}{(1+r)^{\lambda}}\right) \rightarrow 0 \quad \text { as } \nu \rightarrow \infty .
\end{aligned}
$$

$I_{3}(\nu)$ can be expressed as an integral over the whole space:

$$
I_{3}(\nu)=\int_{\mathbb{R}^{3}} \varphi(y)\left(\left|x_{\nu}-y\right|^{\gamma} w\left(x_{\nu}-y\right)-v_{\nu}(y)\right) d y,
$$

where

$$
v_{\nu}: \mathbb{R}^{3} \ni y \mapsto \begin{cases}0 & \text { for } y=x_{\nu} \\ w_{\infty}\left(\left(x_{\nu}-y\right) /\left|x_{\nu}-y\right|\right) & \text { for } y \neq x_{\nu}\end{cases}
$$

According to the assumption (3.7),

$$
\forall y \in \mathbb{R}^{3}: \lim _{\nu \rightarrow \infty}\left(\left|x_{\nu}-y\right|^{\gamma} w\left(x_{\nu}-y\right)-v_{\nu}(y)\right)=0 .
$$

The integrand in $\left(3^{\prime} .11\right)$ is majorized by $|\varphi(y)| \cdot\left(C+\max _{\zeta \in S_{2}}\left|w_{\infty}(\zeta)\right|\right)$, thus by $(\overline{\mathrm{L}}), \lim _{\nu \rightarrow \infty} I_{3}(\nu)=0$.

Keeping the notation $\left(3^{\prime} .12\right)$,we have

$$
I_{4}(\nu)=\int_{\mathbb{R}^{3}} \varphi(y)\left(v_{\nu}(y)-w_{\infty}\left(x_{\nu} /\left|x_{\nu}\right|\right)\right) d y .
$$

It is clear that

$$
\sup _{y \in \mathbb{R}^{3}}\left|v_{\nu}(y)-w_{\infty}\left(x_{\nu} /\left|x_{\nu}\right|\right)\right| \leq 2 \cdot \max _{\zeta \in S_{2}}\left|w_{\infty}(\zeta)\right|,
$$


therefore, in view of $(\overline{\mathrm{L}})$, it suffices to show that

$$
\lim _{\nu \rightarrow \infty}\left(v_{\nu}(y)-w_{\infty}\left(x_{\nu} /\left|x_{\nu}\right|\right)\right)=0, \quad \forall y \in \mathbb{R}^{3} .
$$

For a given $y \in \mathbb{R}^{3}$ there is $\nu_{0} \in \mathbb{N}$ such that $y \neq x_{\nu}$ for $\nu>\nu_{0}$. Let $\varepsilon>0$. The function $w_{\infty}$ is uniformly continuous, thus for some $\delta>0$,

$$
\left|\zeta_{1}-\zeta_{2}\right|<\delta \Rightarrow\left|w_{\infty}\left(\zeta_{1}\right)-w_{\infty}\left(\zeta_{2}\right)\right|<\varepsilon
$$

We have

$$
\lim _{\nu \rightarrow \infty}\left|\frac{x_{\nu}}{\left|x_{\nu}\right|}-\frac{y}{\left|x_{\nu}\right|}\right|=1
$$

since $1-|y| /\left|x_{\nu}\right| \leq\left|x_{\nu} /\right| x_{\nu}|-y /| x_{\nu}|| \leq 1+|y| /\left|x_{\nu}\right|$. Simultaneously

$$
\left|\left(\frac{x_{\nu}}{\left|x_{\nu}\right|} \mid \frac{y}{\left|x_{\nu}\right|}\right)\right| \leq \frac{|y|}{\left|x_{\nu}\right|} \rightarrow 0 \quad \text { as } \nu \rightarrow \infty
$$

therefore

$$
\begin{aligned}
& \left|\frac{x_{\nu}-y}{\left|x_{\nu}-y\right|}-\frac{x_{\nu}}{\left|x_{\nu}\right|}\right|^{2}=2\left(1-\left(1-\left(\frac{x_{\nu}}{\left|x_{\nu}\right|} \mid \frac{y}{\left|x_{\nu}\right|}\right)\right) /\left|\frac{x_{\nu}}{\left|x_{\nu}\right|}-\frac{y}{\left|x_{\nu}\right|}\right|\right) \rightarrow 0 \\
& \text { as } \nu \rightarrow \infty \text {. }
\end{aligned}
$$

In particular,

$$
\exists \nu_{1}>\nu_{0} \forall \nu>\nu_{1}:\left|\frac{x_{\nu}-y}{\left|x_{\nu}-y\right|}-\frac{x_{\nu}}{\left|x_{\nu}\right|}\right|^{2}<\delta^{2}
$$

So

$$
\forall \nu>\nu_{1}:\left|v_{\nu}(y)-w_{\infty}\left(\frac{x_{\nu}}{\left|x_{\nu}\right|}\right)\right|=\left|w_{\infty}\left(\frac{x_{\nu}-y}{\left|x_{\nu}-y\right|}\right)-w_{\infty}\left(\frac{x_{\nu}}{\left|x_{\nu}\right|}\right)\right|<\varepsilon .
$$

In this way we proved $\left(3^{\prime} .13\right)$, and thereby $\left(3^{\prime} .9\right)$ for $k=4$.

It remains to prove $\left(3^{\prime} .9\right)$ for $k=1$. We choose a "buffer" $\Gamma \in$ ] $\max \{3, \gamma, \lambda-1\}, \lambda[$, we define

$$
\begin{gathered}
B_{\nu}:=\left\{y \in \mathbb{R}^{3}:\left|x_{\nu}\right|>1+\left|x_{\nu}-y\right|\right\}, \\
b_{\nu}: \mathbb{R}^{3} \ni y \mapsto \begin{cases}0 & \text { for } y \in B_{\nu}, \\
1-\left(\frac{\left|x_{\nu}\right|}{1+\left|x_{\nu}-y\right|}\right)^{\gamma} & \text { for } y \in \mathbb{R}^{3} \backslash B_{\nu},\end{cases}
\end{gathered}
$$

and we estimate

$$
\left|I_{1}(\nu)\right| \leq C^{2} \int_{\mathbb{R}^{3}}(1+|y|)^{-\lambda}\left|\left(\frac{\left|x_{\nu}\right|}{1+\left|x_{\nu}-y\right|}\right)^{\gamma}-1\right| d y
$$




$$
\begin{aligned}
= & C^{2} \int_{\mathbb{R}^{3} \backslash B_{\nu}}(1+|y|)^{-\lambda}\left(1-\left(\frac{\left|x_{\nu}\right|}{1+\left|x_{\nu}-y\right|}\right)^{\gamma}\right) d y \\
& +C^{2} \int_{B_{\nu}}(1+|y|)^{-\lambda}\left(\left(\frac{\left|x_{\nu}\right|}{1+\left|x_{\nu}-y\right|}\right)^{\Gamma}-1\right) d y \\
\leq & C^{2} \int_{\mathbb{R}^{3}} \frac{b_{\nu}(y)}{(1+|y|)^{\lambda}} d y+C^{2} \int_{B_{\nu}}(1+|y|)^{-\lambda}\left(\left(\frac{\left|x_{\nu}\right|}{1+\left|x_{\nu}-y\right|}\right)^{\Gamma}-1\right) d y .
\end{aligned}
$$

By $(\overline{\mathrm{L}})$ the integral over $\mathbb{R}^{3}$ on the right-hand side of $\left(3^{\prime} .15\right)$ tends to zero, because $\left\|b_{\nu}\right\|_{L^{\infty}} \leq 1$ and, by $\left(3^{\prime} .14\right)$, for $y \in \mathbb{R}^{3}$,

$$
\left|b_{\nu}(y)\right| \leq\left|1-\left(\frac{\left|x_{\nu}\right|}{1+\left|x_{\nu}-y\right|}\right)^{\gamma}\right| \rightarrow 0 \quad \text { as } \nu \rightarrow \infty .
$$

It remains to show that also

$\left(3^{\prime} .16\right)$ the integral over $B_{\nu}$ on the right-hand side of $\left(3^{\prime} .15\right)$ tends to zero as $\nu \rightarrow \infty$.

We have

$$
\begin{aligned}
&\left|x_{\nu}\right| \leq\left|x_{\nu}-y\right|+|y| \leq\left(1+\left|x_{\nu}-y\right|\right)+(1+|y|) \\
& \Rightarrow\left|x_{\nu}\right|-\left(1+\left|x_{\nu}-y\right|\right) \leq 1+|y|
\end{aligned}
$$

Defining $r_{\nu}(y):=\left|x_{\nu}\right| /\left(1+\left|x_{\nu}-y\right|\right)$ for $y \in B_{\nu}$ and bearing in mind $\left(3^{\prime} .17\right)$ we estimate

$$
\begin{aligned}
& \left(\frac{\left|x_{\nu}\right|}{1+\left|x_{\nu}-y\right|}\right)^{\Gamma}-1 \\
& \quad=r_{\nu}(y)^{\Gamma}-1=\int_{1}^{r_{\nu}(y)} \Gamma \cdot t^{\Gamma-1} d t \leq\left(r_{\nu}(y)-1\right) \cdot \Gamma r_{\nu}(y)^{\Gamma-1} \\
& =\Gamma\left|x_{\nu}\right|^{\Gamma-1}\left(1+\left|x_{\nu}-y\right|\right)^{-\Gamma}\left(\left|x_{\nu}\right|-\left(1+\left|x_{\nu}-y\right|\right)\right) \\
& \leq \Gamma\left|x_{\nu}\right|^{\Gamma-1}\left(1+\left|x_{\nu}-y\right|\right)^{-\Gamma}(1+|y|)
\end{aligned}
$$

We can now finish the proof of $\left(3^{\prime} .16\right)$ :

$$
\begin{aligned}
\int_{B_{\nu}}(1+|y|)^{-\lambda} & \left(\left(\frac{\left|x_{\nu}\right|}{1+\left|x_{\nu}-y\right|}\right)^{\Gamma}-1\right) d y \\
\leq & \int_{B_{\nu}}(1+|y|)^{-\lambda} \Gamma\left|x_{\nu}\right|^{\Gamma-1}\left(1+\left|x_{\nu}-y\right|\right)^{-\Gamma}(1+|y|) d y
\end{aligned}
$$




$$
\begin{aligned}
& \leq \Gamma\left|x_{\nu}\right|^{\Gamma-1} \int_{\mathbb{R}^{3}}(1+|y|)^{1-\lambda}\left(1+\left|x_{\nu}-y\right|\right)^{-\Gamma} d y \\
& \leq \Gamma\left(1+\left|x_{\nu}\right|\right)^{\Gamma-1}\left((1+|\cdot|)^{-(\lambda-1)} *(1+|\cdot|)^{-\Gamma}\right)\left(x_{\nu}\right) \\
& \leq \Gamma\left(1+\left|x_{\nu}\right|\right)^{\Gamma-1}\left(1+\left|x_{\nu}\right|\right)^{1-\lambda} C_{\Gamma}(\lambda-1) \\
& =\Gamma C_{\Gamma}(\lambda-1)\left(1+\left|x_{\nu}\right|\right)^{\Gamma-\lambda} \rightarrow 0 \quad \text { as } \nu \rightarrow \infty .
\end{aligned}
$$

The proof of Theorem (3.5) is complete.

\section{$4^{\prime}$. Proofs}

$\left(4^{\prime} .1\right)$ Lemma. For all $r \in \mathbb{R}$,

$$
\begin{gathered}
\sup _{s \in \mathbb{R}} \frac{s}{a+s^{2}}=\frac{1}{2 \sqrt{a}}, \\
\sup _{s \in \mathbb{R}} \frac{a+(r+s)^{2}}{a+s^{2}} \leq 1+\left|\frac{r}{\sqrt{a}}\right|+\left|\frac{r}{\sqrt{a}}\right|^{2}, \\
\sup _{s \in \mathbb{R}} \frac{\left(a+s^{2}\right)^{2}}{\left(a+(s-r)^{2}\right)\left(a+(s+r)^{2}\right)}=1+\frac{r^{2}}{4 a} .
\end{gathered}
$$

Proof. (4'.2) follows from

$$
\frac{d}{d s} \frac{s}{a+s^{2}}=\frac{a-s^{2}}{\left(a+s^{2}\right)^{2}} .
$$

$\left(4^{\prime} .3\right)$ results from $\left(4^{\prime} .2\right)$, because

$$
\frac{a+(r+s)^{2}}{a+s^{2}}=1+r\left(\frac{r}{a+s^{2}}+2 \cdot \frac{s}{a+s^{2}}\right) \leq 1+|r|\left(\frac{|r|}{a}+2 \cdot \frac{1}{2 \sqrt{a}}\right) .
$$

Finally, we prove $\left(4^{\prime} .4\right)$ :

$$
\frac{\left(a+s^{2}\right)^{2}}{\left(a+(s-r)^{2}\right)\left(a+(s+r)^{2}\right)}=\frac{\left(a+s^{2}\right)^{2}}{\left(a+s^{2}\right)^{2}+r^{4}+2 a r^{2}-2 r^{2} s^{2}}=: g(s) .
$$

It follows that $g(\mathbb{R})=f\left(\mathbb{R}_{+}\right)$, where

$$
f(t):=\frac{(a+t)^{2}}{(a+t)^{2}+r^{4}+2 a r^{2}-2 r^{2} t} .
$$

Therefore it is sufficient to find $\sup _{t>0} f(t)$. Just note that $f^{\prime}\left(r^{2}+3 a\right)=0$, $f^{\prime}>0$ on the left of $r^{2}+3 a, f^{\prime}<0$ on the right of $r^{2}+3 a$.

$\left(4^{\prime} .5\right)$ Proof of Lemma (4.1). Fix $t>0$ and $x \in \mathbb{R}^{3}$. In (2.7) we substitute

$$
\kappa(r)=(2 \nu t)^{-3 / 2} \exp \left(-\frac{1}{2}\left(\frac{r}{\sqrt{2 \nu t}}\right)^{2}\right)
$$

and we estimate, using $\left(4^{\prime} .4\right)$, 


$$
\begin{aligned}
(2 \pi)^{-3 / 2} & \left(E(t, \cdot) * \frac{1}{\left(a+|\cdot|^{2}\right)^{2}}\right)(x) \\
& =(2 \pi)^{-3 / 2}\left(\kappa(|\cdot|) * \frac{1}{\left(a^{2}+|\cdot|^{2}\right)^{2}}\right)(x) \\
& =\left(\frac{2}{\pi}\right)^{1 / 2} \int_{0}^{\infty} \frac{r^{2} \kappa(r) d r}{\left(a+(r-|x|)^{2}\right)\left(a+(r+|x|)^{2}\right)} \\
& \leq\left(\frac{2}{\pi}\right)^{1 / 2} \int_{0}^{\infty} \frac{r^{2} \kappa(r)}{\left(a+|x|^{2}\right)^{2}}\left(1+\frac{r^{2}}{4 a}\right) d r \\
& =\left(\frac{2}{\pi}\right)^{1 / 2} \int_{0}^{\infty}\left(s^{2}+\frac{\nu t s^{4}}{2 a}\right) \cdot(2 \nu t)^{3 / 2} \kappa(\sqrt{2 \nu t} s) d s \frac{1}{\left(a+|x|^{2}\right)^{2}} \\
& =\left(\frac{2}{\pi}\right)^{1 / 2}\left(\int_{0}^{\infty} s^{2} e^{-s^{2} / 2} d s+\frac{\nu t}{2 a} \int_{0}^{\infty} s^{4} e^{-s^{2} / 2} d s\right) \frac{1}{\left(a+|x|^{2}\right)^{2}}
\end{aligned}
$$

$\left(4^{\prime} .6\right)$ Proof of Lemma (4.2). Fix $\tau>0$ and $x \in \mathbb{R}^{3} . F(\tau, \cdot)$ is a radius function; namely, $F(\tau, y)=(2 \nu \tau)^{-5 / 2} \kappa(|y|)$, where

$$
\kappa(r):= \begin{cases}r & \text { for } r<\sqrt{2 \nu \tau} \\ 0 & \text { for } r \geq \sqrt{2 \nu \tau}\end{cases}
$$

We differentiate the equality (obtained with the aid of (2.7))

$$
\frac{1}{4 \pi}\left(\left(a+|\cdot|^{2}\right)^{-2} * F(\tau, \cdot)\right)(x)=(2 \nu \tau)^{-5 / 2} \int_{0}^{\infty} \frac{r^{2} \kappa(r) d r}{\left(a+(r-|x|)^{2}\right)\left(a+(r+|x|)^{2}\right)}
$$

twice with respect to $a$ and we get

$$
\begin{aligned}
((a & \left.\left.+|\cdot|^{2}\right)^{-4} * F(\tau, \cdot)\right)(x) \\
& =\frac{2 \pi}{3}(2 \nu \tau)^{-5 / 2} \int_{0}^{\sqrt{2 \nu \tau}} r^{3} \frac{d^{2}}{d a^{2}} \frac{1}{\left(a+(r-|x|)^{2}\right)\left(a+(r+|x|)^{2}\right)} d r .
\end{aligned}
$$

For $z_{1}, z_{2} \in \mathbb{R}_{+}$we compute

$$
\frac{d^{2}}{d a^{2}} \frac{1}{\left(a+z_{1}\right)\left(a+z_{2}\right)}=2 \cdot \frac{3 a \cdot\left(a+z_{1}+z_{2}\right)+z_{1}^{2}+z_{2}^{2}+z_{1} z_{2}}{\left(a+z_{1}\right)^{3}\left(a+z_{2}\right)^{3}} .
$$

In particular, if, for a fixed $r>0, z_{1}:=(r-|x|)^{2}, z_{2}:=(r+|x|)^{2}$ then

$$
\begin{gathered}
z_{1}+z_{2}=2 r^{2}+2|x|^{2}, \quad z_{1}^{2}+z_{2}^{2}=2 r^{4}+2|x|^{4}+12 r^{2}|x|^{2} \\
z_{1} z_{2}=r^{4}-2 r^{2}|x|^{2}+|x|^{4}
\end{gathered}
$$


After substituting $\left(4^{\prime} .9\right)$ in $\left(4^{\prime} .8\right)$ we estimate

$$
\begin{aligned}
\frac{d^{2}}{d a^{2}} \frac{1}{\left(a+(r-|x|)^{2}\right)\left(a+(r+|x|)^{2}\right)} \\
=2 \frac{3\left(a+r^{2}+|x|^{2}\right)^{2}+4 r^{2}|x|^{2}}{\left(a+(r-|x|)^{2}\right)^{3}\left(a+(r+|x|)^{2}\right)^{3}} \\
=\frac{2}{\left(a+(r-|x|)^{2}\right)^{3}\left(a+(r+|x|)^{2}\right)} \\
\\
\cdot \frac{3\left(a+r^{2}+|x|^{2}\right)^{2}+4 r^{2}|x|^{2}}{\left(a+r^{2}+|x|^{2}\right)^{2}+4 r^{2}|x|^{2}+4 r|x|\left(a+r^{2}+|x|^{2}\right)} \\
\leq \frac{2}{\left(a+(r-|x|)^{2}\right)^{3}\left(a+(r+|x|)^{2}\right)} \cdot 3 .
\end{aligned}
$$

If, additionally, $r<\sqrt{2 \nu \tau}$ then after applying $\left(4^{\prime} .3\right)$ and $\left(4^{\prime} .4\right)$ we have

$$
\begin{aligned}
& \frac{d^{2}}{d a^{2}} \frac{1}{\left(a+(r-|x|)^{2}\right)\left(a+(r+|x|)^{2}\right)} \\
\leq & \frac{6}{\left(a+|x|^{2}\right)^{4}}\left(\frac{a+(r+|x|)^{2}}{a+|x|^{2}}\right)^{2}\left(\frac{\left(a+|x|^{2}\right)^{2}}{\left(a+(|x|-r)^{2}\right)\left(a+(|x|+r)^{2}\right)}\right)^{3} \\
\leq & \frac{6}{\left(a+|x|^{2}\right)^{4}}\left(1+\frac{r}{\sqrt{a}}+\left(\frac{r}{\sqrt{a}}\right)^{2}\right)^{2}\left(1+\frac{r^{2}}{4 a}\right)^{3} \\
\leq & \frac{6}{\left(a+|x|^{2}\right)^{4}}\left(1+\sqrt{\frac{2 \nu \tau}{a}}+\frac{2 \nu \tau}{a}\right)^{2}\left(1+\frac{\nu \tau}{2 a}\right)^{3} .
\end{aligned}
$$

Using ( $\left.4^{\prime} .11\right)$ we estimate the right-hand side of $\left(4^{\prime} .7\right)$ to get

$$
\begin{aligned}
& \left(\left(a+|\cdot|^{2}\right)^{-4} * F(\tau, \cdot)\right)(x) \\
& \quad \leq(2 \nu \tau)^{-1 / 2} \pi\left(1+\sqrt{\frac{2 \nu \tau}{a}}+\frac{2 \nu \tau}{a}\right)^{2}\left(1+\frac{\nu \tau}{2 a}\right)^{3}\left(a+|x|^{2}\right)^{-4} .
\end{aligned}
$$

We put

$$
M(\varrho)=2^{-1 / 2} \pi(1+\sqrt{2 \varrho}+2 \varrho)^{2}\left(1+\frac{1}{2} \varrho\right)^{3} .
$$

$\left(4^{\prime} .13\right)$ Proof of Lemma (4.3). Fix $\tau>0$ and $x \in \mathbb{R}^{3}$. Then $G(\tau, \cdot)$ $=\kappa(|\cdot|)$, where

$$
\kappa(r):= \begin{cases}0 & \text { if } r<\sqrt{2 \nu \tau} \\ r^{-4} & \text { if } r \geq \sqrt{2 \nu \tau} .\end{cases}
$$

We differentiate the equality (obtained from (2.7))

$$
\frac{1}{4 \pi}\left(\left(a+|\cdot|^{2}\right)^{-2} * G(\tau, \cdot)\right)(x)=\int_{0}^{\infty} \frac{r^{2} \kappa(r) d r}{\left(a+(r-|x|)^{2}\right)\left(a+(r+|x|)^{2}\right)}
$$


twice with respect to $a$ and next, using $\left(4^{\prime} .10\right)$ and $\left(4^{\prime} .4\right)$, we estimate

$\left(4^{\prime} .14\right) \quad\left(\left(a+|\cdot|^{2}\right)^{-4} * G(\tau, \cdot)\right)(x)$

$$
\begin{aligned}
& =\frac{2 \pi}{3} \int_{\sqrt{2 \nu \tau}}^{\infty} r^{-2} \frac{d^{2}}{d a^{2}} \frac{1}{\left(a+(r-|x|)^{2}\right)\left(a+(r+|x|)^{2}\right)} d r \\
& \leq \frac{4 \pi}{\left(a+|x|^{2}\right)^{2}} \int_{\sqrt{2 \nu \tau}}^{\infty} \frac{r^{-2}}{\left(a+(r-|x|)^{2}\right)^{2}} \cdot \frac{\left(a+|x|^{2}\right)^{2}}{\left(a+(|x|-r)^{2}\right)\left(a+(|x|+r)^{2}\right)} d r \\
& \leq \frac{4 \pi}{\left(a+|x|^{2}\right)^{2}}\left(\int_{\sqrt{2 \nu \tau}}^{\infty} \frac{r^{-2} d r}{\left(a+(r-|x|)^{2}\right)^{2}}+\frac{1}{4 a} \int_{\sqrt{2 \nu \tau}}^{\infty} \frac{d r}{\left(a+(r-|x|)^{2}\right)^{2}}\right) .
\end{aligned}
$$

We differentiate the formula

$$
\int\left(a+(r-|x|)^{2}\right)^{-1} d r=a^{-1 / 2} \arctan \left((r-|x|) \cdot a^{-1 / 2}\right)
$$

with respect to $a$ and we get

$$
\int\left(a+(r-|x|)^{2}\right)^{-2} d r=\frac{1}{2} \cdot a^{-3 / 2}\left(\arctan \frac{r-|x|}{\sqrt{a}}+\sqrt{a} \cdot \frac{r-|x|}{a+(r-|x|)^{2}}\right) .
$$

Hence, after applying $\left(4^{\prime} .2\right)$, we obtain

$$
\begin{aligned}
& \int_{\sqrt{2 \nu \tau}}^{\infty} \frac{d r}{\left(a+(r-|x|)^{2}\right)^{2}} \\
& =\frac{1}{2} \cdot a^{-3 / 2}\left(\frac{\pi}{2}+\arctan \frac{|x|-\sqrt{2 \nu \tau}}{\sqrt{a}}+a^{1 / 2} \cdot \frac{|x|-\sqrt{2 \nu \tau}}{a+(|x|-\sqrt{2 \nu \tau})^{2}}\right) \\
& \leq \frac{1}{2} a^{-3 / 2}\left(\frac{\pi}{2}+\frac{\pi}{2}+\sqrt{a} \cdot \frac{1}{2 \sqrt{a}}\right)=\frac{1}{4} \cdot(2 \pi+1) \cdot a^{-3 / 2} .
\end{aligned}
$$

With the aid of the formula

$$
\begin{aligned}
& \int \frac{r^{-2} d r}{a+(r-|x|)^{2}} \\
& =-\frac{1}{a+|x|^{2}}\left(\frac{|x|}{a+|x|^{2}} \ln \frac{a+(r-|x|)^{2}}{r^{2}}+\frac{1}{r}+\frac{a-|x|^{2}}{a+|x|^{2}} \frac{1}{\sqrt{a}} \arctan \frac{r-|x|}{\sqrt{a}}\right)
\end{aligned}
$$

we compute

$$
\int_{\sqrt{2 \nu \tau}}^{\infty} \frac{r^{-2} d r}{a+(r-|x|)^{2}}=(2 \nu \tau)^{-1 / 2}\left(a+|x|^{2}\right)^{-1}
$$

$+\left(a+|x|^{2}\right)^{-2}\left(|x| \ln \frac{a+(|x|-\sqrt{2 \nu \tau})^{2}}{2 \nu \tau}-\frac{a-|x|^{2}}{\sqrt{a}}\left(\frac{\pi}{2}+\arctan \frac{|x|-\sqrt{2 \nu \tau}}{\sqrt{a}}\right)\right)$.

We differentiate $\left(4^{\prime} .16\right)$ with respect to $a$, and next, using the inequalities 
$\ln s<s^{1 / 2}$ for $s>0$ (because $\left.e>2\right),\left(4^{\prime} .2\right)$ and $\left(4^{\prime} .3\right)$, we estimate

$$
\begin{aligned}
& \int_{\sqrt{2 \nu \tau}}^{\infty} \frac{r^{-2} d r}{\left(a+(r-|x|)^{2}\right)^{2}} \\
& =\frac{2}{\sqrt{a}} \cdot \frac{1}{\left(a+|x|^{2}\right)^{2}} \cdot \frac{|x|^{2}-a}{|x|^{2}+a}\left(\frac{\pi}{2}+\arctan \frac{|x|-\sqrt{2 \nu \tau}}{\sqrt{a}}\right) \\
& +\frac{a^{-3 / 2}}{2} \cdot \frac{1}{a+|x|^{2}} \cdot\left(\frac{\pi}{2}+\arctan \frac{|x|-\sqrt{2 \nu \tau}}{\sqrt{a}}\right) \\
& +\frac{1}{2 a} \cdot \frac{1}{a+|x|^{2}} \cdot \frac{|x|^{2}-a}{|x|^{2}+a} \cdot \frac{|x|-\sqrt{2 \nu \tau}}{a+(|x|-\sqrt{2 \nu \tau})^{2}} \\
& +\frac{(2 \nu \tau)^{-1 / 2}}{\left(a+|x|^{2}\right)^{2}}+\frac{2|x|}{\left(a+|x|^{2}\right)^{3}} \ln \frac{a+(|x|-\sqrt{2 \nu \tau})^{2}}{2 \nu \tau} \\
& -\frac{|x|}{\left(a+|x|^{2}\right)^{2}} \cdot \frac{1}{a+(|x|-\sqrt{2 \nu \tau})^{2}} \\
& \leq \frac{2}{\sqrt{a}} \cdot \frac{1}{a^{2}} \cdot 1 \cdot \pi+\frac{a^{-3 / 2}}{2} \cdot \frac{1}{a} \cdot \pi \\
& +\frac{1}{2 a} \cdot \frac{1}{a+|x|^{2}} \cdot\left|\frac{|x|^{2}-a}{|x|^{2}+a}\right| \cdot\left|\frac{|x|-\sqrt{2 \nu \tau}}{a+(|x|-\sqrt{2 \nu \tau})^{2}}\right| \\
& +\frac{(2 \nu \tau)^{-1 / 2}}{\left(a+|x|^{2}\right)^{2}}+\frac{2}{\left(a+|x|^{2}\right)^{2}} \cdot \frac{|x|}{\sqrt{a+|x|^{2}}} \cdot\left(\frac{1}{a+|x|^{2}}\right)^{1 / 2} \\
& \cdot\left(\frac{a+(|x|-\sqrt{2 \nu \tau})^{2}}{2 \nu \tau}\right)^{1 / 2}+0 \\
& \leq \frac{5}{2} \pi a^{-5 / 2}+\frac{1}{2 a} \cdot \frac{1}{a} \cdot 1 \cdot \frac{1}{2 \sqrt{a}}+\frac{(2 \nu \tau)^{-1 / 2}}{\left(a+|x|^{2}\right)^{2}} \\
& +\frac{2}{\left(a+|x|^{2}\right)^{2}} \cdot 1 \cdot\left(\frac{1}{2 \nu \tau}\right)^{1 / 2}\left(\frac{a+(|x|-\sqrt{2 \nu \tau})^{2}}{a+|x|^{2}}\right)^{1 / 2} \\
& \leq \frac{1}{4} \cdot(10 \pi+1) \cdot a^{-5 / 2} \\
& +(2 \nu \tau)^{-1 / 2}\left(1+2\left(1+\sqrt{\frac{2 \nu \tau}{a}}+\frac{2 \nu \tau}{a}\right)^{1 / 2}\right)\left(a+|x|^{2}\right)^{-2} .
\end{aligned}
$$

Combining ( $\left.4^{\prime} .17\right)$ and $\left(4^{\prime} .15\right)$ allows us to conclude the estimate $\left(4^{\prime} .14\right)$ :

$$
\begin{aligned}
& \left(\left(a+|\cdot|^{2}\right)^{-4} * G(\tau, \cdot)\right)(x) \leq \frac{\pi}{4} \cdot(42 \pi+5) \cdot a^{-5 / 2}\left(a+|x|^{2}\right)^{-2} \\
& \quad+(\nu \tau)^{-1 / 2} \cdot 2 \pi \sqrt{2}\left(1+2\left(1+\sqrt{\frac{2 \nu \tau}{a}}+\frac{2 \nu \tau}{a}\right)^{1 / 2}\right)\left(a+|x|^{2}\right)^{-4} .
\end{aligned}
$$


We put

$$
\begin{gathered}
C=\frac{\pi}{4} \cdot(42 \pi+5), \\
M(\varrho)=2 \pi \sqrt{2}\left(1+2(1+\sqrt{2 \varrho}+2 \varrho)^{1 / 2}\right) .
\end{gathered}
$$

Acknowledgments. Support by the Polish State Committee for Scientific Research in the course of this work is acknowledged with gratitude (project no. 3366292 03, financed during the period 1992-1993).

\section{References}

[1] K. Holly, Navier-Stokes equations in $\mathbb{R}^{3}$ as a system of nonsingular integral equations of Hammerstein type. An abstract approach, Univ. Iagel. Acta Math. 28 (1991), 151-161.

[2] -, Navier-Stokes equations in $\mathbb{R}^{3}$ : relations between pressure and velocity, Internat. Conf. "Nonlinear Differential Equations", Varna 1987, unpublished.

[3] N. S. Landkof, Foundations of Modern Potential Theory, Nauka, Moscow, 1966 (in Russian).

[4] M. Riesz, Intégrales de Riemann-Liouville et potentiels, Acta Sci. Math. (Szeged) 9 (1938), 1-42.

INSTITUTE OF MATHEMATICS

JAGIELLONIAN UNIVERSITY

REYMONTA 4

30-059 KRAKÓW, POLAND 